Annals of Mathematics 182 (2015), 127-172

http://dx.doi.org/10.4007/annals.2015.182.1.3

\title{
Anomalous dissipation for 1/5-Hölder Euler flows
}

\author{
By Tristan Buckmaster, Camillo De Lellis, Philip Isett, \\ and LÁszló SzÉKELYHIDI, JR.
}

\begin{abstract}
Recently the second and fourth authors developed an iterative scheme for obtaining rough solutions of the $3 \mathrm{D}$ incompressible Euler equations in Hölder spaces. The motivation comes from Onsager's conjecture. The construction involves a superposition of weakly interacting perturbed Beltrami flows on infinitely many scales. An obstruction to better regularity arises from the errors in the linear transport of a fast periodic flow by a slow velocity field.

In a recent paper the third author has improved upon the methods, introducing some novel ideas on how to deal with this obstruction, thereby reaching a better Hölder exponent - albeit weaker than the one conjectured by Onsager. In this paper we give a shorter proof of this final result, adhering more to the original scheme of the second and fourth authors and introducing some new devices. More precisely we show that for any positive $\varepsilon$, there exist periodic solutions of the $3 \mathrm{D}$ incompressible Euler equations that dissipate the total kinetic energy and belong to the Hölder class $C^{1 / 5-\varepsilon}$.
\end{abstract}

\section{Introduction}

In what follows $\mathbb{T}^{3}$ denotes the 3 -dimensional torus, i.e., $\mathbb{T}^{3}=\mathbb{S}^{1} \times \mathbb{S}^{1} \times \mathbb{S}^{1}$. In this note we give a proof of the following theorem.

TheOREm 0.1. Assume $e:[0,1] \rightarrow \mathbb{R}$ is a positive smooth function and $\varepsilon$ a positive number. Then there are a continuous vector field $v \in C^{1 / 5-\varepsilon}\left(\mathbb{T}^{3} \times\right.$ $\left.[0,1], \mathbb{R}^{3}\right)$ and a continuous scalar field $p \in C^{2 / 5-2 \varepsilon}\left(\mathbb{T}^{3} \times[0,1], \mathbb{R}\right)$ that solve the incompressible Euler equations

$$
\left\{\begin{array}{l}
\partial_{t} v+\operatorname{div}(v \otimes v)+\nabla p=0, \\
\operatorname{div} v=0
\end{array}\right.
$$

in the sense of distributions and such that

$$
e(t)=\int|v|^{2}(x, t) d x \quad \forall t \in[0,1] .
$$

(C) 2015 Department of Mathematics, Princeton University. 
Results of this type are associated with the famous conjecture of Onsager. In a nutshell, the question is about whether or not weak solutions in a given regularity class satisfy the law of kinetic energy conservation or not. For classical solutions (say, $v \in C^{1}$ ), we can multiply (1) by $v$ itself, integrate by parts and obtain the energy balance

$$
\int_{\mathbb{T}^{3}}|v(x, t)|^{2} d x=\int_{\mathbb{T}^{3}}|v(x, 0)|^{2} d x \quad \text { for all } t>0 .
$$

On the other hand, for weak solutions (say, merely $v \in L^{2}$ ) (3) might be violated, and this possibility has been considered for a rather long time in the context of 3-dimensional turbulence. In his famous note [21] about statistical hydrodynamics, Onsager considered weak solutions satisfying the Hölder condition

$$
\left|v(x, t)-v\left(x^{\prime}, t\right)\right| \leq C\left|x-x^{\prime}\right|^{\theta},
$$

where the constant $C$ is independent of $x, x^{\prime} \in \mathbb{T}^{3}$ and $t$. He conjectured that

(a) Any weak solution $v$ satisfying (4) with $\theta>\frac{1}{3}$ conserves the energy;

(b) For any $\theta<\frac{1}{3}$, there exist weak solutions $v$ satisfying (4) that do not conserve the energy.

This conjecture is also very closely related to Kolmogorov's famous K41 theory [19] for homogeneous isotropic turbulence in 3 dimensions. We refer the interested reader to [15], [22], [14]. Part (a) of the conjecture is by now fully resolved: it was first considered by Eyink in [13] following Onsager's original calculations and proved by E. Constantin and Titi in [3]. Slightly weaker assumptions on $v$ (in Besov spaces) were subsequently shown to be sufficient for energy conservation in [12], [2].

In this paper we are concerned with part (b) of the conjecture. Weak solutions violating the energy equality have been constructed for a long time, starting with the seminal work of Scheffer [23] and Shnirelman [24]. In [8], [9] a new point of view was introduced, relating the issue of energy conservation to Gromov's $h$-principle; see also [10]. In [11] and [7] the first constructions of continuous and Hölder-continuous weak solutions violating the energy equality appeared. In particular, in [7] the authors proved Theorem 0.1 with Hölder exponent $1 / 10-\varepsilon$ (replacing the exponent $1 / 5-\varepsilon$ in this paper).

The threshold exponent $\frac{1}{5}$ has been recently reached by the third author in [18] (although strictly speaking the result of [18] is a variant of Theorem 0.1, since it shows the existence of nontrivial solutions that are compactly supported in time, rather than prescribing the total kinetic energy). Our aim in this note is to give a shorter proof of this improvement in the Hölder exponent and isolate the main new ideas of [18] compared to [11], [7]. We observe in passing that the arguments given here can be easily modified to produce nontrivial 
solutions with compact support in time but losing control on the exact shape of the energy. The question of producing a solution matching an energy profile $e$ that might vanish is subtler. A similar issue has been recently treated in the paper [6].

0.1. Euler-Reynolds system and the convex integration scheme. Let us now outline the principal ideas of the present scheme. The scheme will be based on the constructions given in [11], [7]. We will also make use of some of the novel ideas introduced in [18].

The proof is achieved through an iteration scheme. At each step $q \in \mathbb{N}$ we construct a triple $\left(v_{q}, p_{q}, \stackrel{\circ}{R}_{q}\right)$ solving the Euler-Reynolds system (see [11, Def. 2.1]):

$$
\left\{\begin{array}{l}
\partial_{t} v_{q}+\operatorname{div}\left(v_{q} \otimes v_{q}\right)+\nabla p_{q}=\operatorname{div} \stackrel{\circ}{R}_{q}, \\
\operatorname{div} v_{q}=0 .
\end{array}\right.
$$

The $3 \times 3$ symmetric traceless tensor $\stackrel{\circ}{R}_{q}$ is related to the so-called Reynolds stress, a quantity that arises naturally when considering highly oscillatory solutions of the Euler equations or, equivalently, when taking weak limits of solutions of (1). (We refer the reader to [10] for a thorough discussion.)

The size of the perturbation

$$
w_{q}:=v_{q}-v_{q-1}
$$

will be measured by two parameters: $\delta_{q}^{1 / 2}$ is the amplitude and $\lambda_{q}$ the frequency. More precisely, denoting the (spatial) Hölder norms by $\|\cdot\|_{k}$ (see Section C for precise definitions),

$$
\begin{aligned}
\left\|w_{q}\right\|_{0} & \leq M \delta_{q}^{1 / 2} \\
\left\|w_{q}\right\|_{1} & \leq M \delta_{q}^{1 / 2} \lambda_{q}
\end{aligned}
$$

and similarly,

$$
\begin{aligned}
& \left\|p_{q}-p_{q-1}\right\|_{0} \leq M^{2} \delta_{q}, \\
& \left\|p_{q}-p_{q-1}\right\|_{1} \leq M^{2} \delta_{q} \lambda_{q},
\end{aligned}
$$

where $M$ is a constant depending only on the function $e=e(t)$ in the theorem.

In constructing the iteration, the new perturbation $w_{q}$ will be chosen so as to balance the previous Reynolds error $\stackrel{\circ}{R}_{q-1}$ in the sense that (cf. equation (5)) we have $\left\|w_{q} \otimes w_{q}\right\|_{0} \sim\left\|\stackrel{\circ}{R}_{q-1}\right\|_{0}$. To make this possible, we then inductively claim the estimates

$$
\begin{aligned}
& \|\stackrel{\circ}{R}\|_{q} \leq \eta \delta_{q+1}, \\
& \left\|\stackrel{\circ}{R}_{q}\right\|_{1} \leq M \delta_{q+1} \lambda_{q},
\end{aligned}
$$


where $\eta$ will be a small constant, again only depending on $e=e(t)$ in the theorem.

Along the iteration we will have

$$
\delta_{q} \rightarrow 0 \quad \text { and } \quad \lambda_{q} \rightarrow \infty
$$

at a rate that is at least exponential. On the one hand (6), (8) and (10) will imply the convergence of the sequence $v_{q}$ to a continuous weak solution of the Euler equations. On the other hand, the precise dependence of $\lambda_{q}$ on $\delta_{q}$ will determine the critical Hölder regularity. Finally, equation (2) will be ensured by

$$
\left.\left|e(t)\left(1-\delta_{q+1}\right)-\int\right| v_{q}\right|^{2}(x, t) d x \mid \leq \frac{1}{4} \delta_{q+1} e(t) .
$$

Note that, being an expression quadratic in $v_{q}$, this estimate is consistent with (10).

Estimates of type (6)-(11) appear already in the paper [7]: although the bound claimed for $\stackrel{\circ}{R}_{q}$ was $\left\|\stackrel{\circ}{R}_{q}\right\|_{1} \leq M \delta_{q}^{1 / 2} \lambda_{q}$, which had been done for ease of notation (cf. [7, Prop 2.2]: $\lambda_{q}$ here corresponds to $\left(D \delta / \bar{\delta}^{2}\right)^{1+\varepsilon}$ there), the actual bound achieved in the proof does in fact correspond to (11) (cf. Step 4 in Section 9). Using (6)-(11) one can obtain some improvement on the exponent $\frac{1}{10}$ from the same construction. However, this improvement is of fairly limited interest, in particular, because the frequencies are still required to grow at a much greater than exponential rate, which is a basic obstruction to higher regularity that was only overcome in [18] using new ideas, which we will describe below.

As for the explicit form of the perturbation, it will consist essentially of a finite sum of modulated Beltrami modes (see Section 1 below), so that

$$
w_{q}(x, t)=\sum_{k} a_{k}(x, t) \phi_{k}(x, t) B_{k} e^{i \lambda_{q} k \cdot x},
$$

where $a_{k}$ is the amplitude, $\phi_{k}$ is a phase function (i.e., $\left|\phi_{k}\right|=1$ ) and $B_{k} e^{i \lambda_{q} k \cdot x}$ is a complex Beltrami mode at frequency $\lambda_{q}$. In fact, a lower order correction to this Ansatz is needed in order to ensure that $w_{q}$ is divergence free.

Next, the Reynolds stress $\stackrel{\circ}{R}_{q}$ and the pressure $p_{q}$ are chosen so that (5) holds. In particular, since (5) is linear in the Reynolds stress, this can be achieved by separately solving the three equations

$$
\begin{aligned}
& \operatorname{div} R-\nabla p_{q}=\operatorname{div}\left(w_{q} \otimes w_{q}+\stackrel{\circ}{R}_{q-1}\right)-\nabla p_{q-1}, \\
& \operatorname{div} R=\partial_{t} w_{q}+v_{q-1} \cdot \nabla w_{q}, \\
& \operatorname{div} R=w_{q} \cdot \nabla v_{q-1}
\end{aligned}
$$

(although the actual decomposition used in the proof is more complicated). The most involved part of the scheme is then to show that such equations can be solved while maintaining the estimates (10)-(11). 
Having a perturbation of the form (13) ensures that the "oscillation part of the error"

$$
\operatorname{div}\left(w_{q} \otimes w_{q}+\stackrel{\circ}{R}_{q-1}\right)
$$

can be absorbed into the pressure; see [11]. (In [18] this term is called "highhigh interference term.")

The main analytical part of the argument goes into choosing $a_{k}$ and $\phi_{k}$ correctly in order to deal with the so-called transport part of the error

$$
\partial_{t} w_{q}+v_{q-1} \cdot \nabla w_{q}
$$

In [11], [7] a second large parameter $\mu\left(=\mu_{q}\right)$ was introduced to deal with this term. In some sense the role of $\mu$ is to interpolate between errors of order 1 in the transport term and errors of order $\lambda_{q}^{-1}$ in the oscillation term.

The approach of [18] begins in parallel with an ansatz for the correction that allows for nonlinear phase functions $\xi_{I}(t, x)$

$$
w_{q}(x, t)=\sum_{I} e^{i \lambda_{q} \xi_{I}(t, x)} v_{I}(x, t),
$$

where here $v_{I}$ plays a similar role to the vector fields $a_{k} B_{k}$ given in (13). ${ }^{1}$ Substituting this ansatz into the equation leads to a transport equation for the phase functions $\xi_{I}(t, x)$ in a way similar to the appearance of Hamilton-Jacobi equations in geometric optics. However, in order to control the high-frequency terms that occur in (14), it is necessary to introduce sharp time cutoffs that restrict the lifespan of the oscillatory waves to time intervals where the phase functions $\xi_{I}$ remain linear and the correction remains close to the form of (13). (The application of time cutoffs is comparable to the use of CFL conditions (cf. [5]) employed in numerical analysis to study evolutionary equations; see Remark 2.)

The construction in [18] can therefore be viewed as well in terms of the Ansatz (13), but with two more ingredients compared to [11], [7], which adhere more closely to the transport structure of the equation. First, the phase functions $\phi_{k}$ are defined using the flow map of the vector field $v_{q}$, whereas in [11], [7] they were functions of $v_{q}$ itself. With the latter choice, the threshold $1 / 5$ seems beyond reach. Secondly, a new set of estimates, complementing, (6)-(12), are introduced. Their purpose is to control the advective derivative of the Reynolds error:

$$
\left\|\partial_{t} \stackrel{\circ}{R}_{q}+v_{q} \cdot \nabla \stackrel{\circ}{R}_{q}\right\|_{0} \leq \delta_{q+1} \delta_{q}^{1 / 2} \lambda_{q} .
$$

\footnotetext{
${ }^{1}$ Note, however, that unlike the vector field $a_{k} B_{k}$, the direction of $v_{I}$ is allowed to vary in spacetime.
} 
This bound implies that the advective derivative of $\stackrel{\circ}{R}_{q}$ must satisfy a better estimate compared to the bounds that hold for either pure spatial or pure temporal derivatives. Maintaining this additional control becomes more involved, as it is important to construct the amplitudes $a_{k}$ in a way that is compatible with the transport structure, and also to take advantage of the bounds for the pressure and transport estimates from the Euler-Reynolds system to close the argument. These ingredients also play a key role in the proof of Theorem 0.1 given here; however, compared to [18], we improve upon the simplicity of their implementation. For instance, since the perturbations in [18] use a nonlinear phase rather than the simple stationary flows used here, a "microlocal" version of the Beltrami flows is needed. This also leads to the necessity of appealing to nonlinear stationary phase lemmas - whereas in the present work linear stationary phase lemmas suffice.

Our purpose here is to show that, although the other ideas exploited in [18] are of independent interest and might also, in principle, lead to better bounds in the future, with the additional control in (15), a scheme much more similar to the one introduced in [11] provides a substantially shorter proof of Theorem 0.1. To this end, we introduce some new devices that greatly simplify the relevant estimates:

(a) We regularize the maps $v_{q}$ and $\stackrel{\circ}{R}_{q}$ in space only and then solve locally in time the free-transport equation in order to approximate $\stackrel{\circ}{R}_{q}$.

(b) Our maps $a_{k}$ are then elementary algebraic functions of the approximation of $\stackrel{\circ}{R}_{q}$.

(c) The estimates for the Reynolds stress are still carried on based on simple stationary "linear" phase arguments.

(d) The proof of (15) is simplified by one commutator estimate that, in spite of having a classical flavor, deals efficiently with one important error term.

Analogously to the scheme presented in [18], we also employ time cutoffs in order to restrict the lifespan of the oscillatory waves. For comparison, it is worth noting that there is a rough correspondence between the family of parameters $\left(\lambda_{q}, \delta_{q}, \delta_{q+1}, \lambda_{q+1}, \mu\right)$ and the parameters $\left(\Xi, e_{v}, e_{R}, N \Xi, \tau^{-1}\right)$ employed in [18]. As we go along, we will also make further references and comparisons to parallel aspects of the proof in [18].

0.2. The main iteration proposition and the proof of Theorem 0.1. Having outlined the general idea above, we proceed with the iteration, starting with the trivial solution $\left(v_{0}, p_{0}, \stackrel{\circ}{R}_{0}\right)=(0,0,0)$. We will construct new triples $\left(v_{q}, p_{q}, \stackrel{\circ}{R}_{q}\right)$ inductively, assuming the estimates (6)-(15).

Proposition 0.2. There are positive constants $M$ and $\eta$ depending only on e such that the following holds. For every $c>\frac{5}{2}$ and $b>1$, if a is sufficiently 
large, then there is a sequence of triples $\left(v_{q}, p_{q}, \stackrel{\circ}{R}_{q}\right)$ starting with $\left(v_{0}, p_{0}, \stackrel{\circ}{R}_{0}\right)=$ $(0,0,0)$, solving (5) and satisfying the estimates (6)-(15), where $\delta_{q}:=a^{-b^{q}}$, $\lambda_{q} \in\left[a^{c b^{q+1}}, 2 a^{c b^{q+1}}\right]$ for $q=0,1,2, \ldots$. In addition, we claim the estimates

$$
\left\|\partial_{t}\left(v_{q}-v_{q-1}\right)\right\|_{0} \leq C \delta_{q}^{1 / 2} \lambda_{q} \quad \text { and } \quad\left\|\partial_{t}\left(p_{q}-p_{q-1}\right)\right\|_{0} \leq C \delta_{q} \lambda_{q}
$$

Proof of Theorem 0.1. Choose any $c>\frac{5}{2}$ and $b>1$, and let $\left(v_{q}, p_{q}, \stackrel{\circ}{R}_{q}\right)$ be a sequence as in Proposition 0.2. It follows then easily that $\left\{\left(v_{q}, p_{q}\right)\right\}$ converge uniformly to a pair of continuous functions $(v, p)$ such that (1) and (2) hold. We introduce the notation $\|\cdot\|_{C \vartheta}$ for Hölder norms in space and time. From (6)-(9), (16) and interpolation, we conclude

$$
\begin{aligned}
\left\|v_{q+1}-v_{q}\right\|_{C^{\vartheta}} & \leq M \delta_{q+1}^{1 / 2} \lambda_{q+1}^{\vartheta} \leq C a^{b^{q+1}(2 c b \vartheta-1) / 2}, \\
\left\|p_{q+1}-p_{q}\right\|_{C^{2 \vartheta}} & \leq M^{2} \delta_{q+1} \lambda_{q+1}^{2 \vartheta} \leq C a^{b^{q+1}(2 c b \vartheta-1)} .
\end{aligned}
$$

Thus, for every $\vartheta<\frac{1}{2 b c}, v_{q}$ converges in $C^{\vartheta}$ and $p_{q}$ in $C^{2 \vartheta}$.

0.3. Plan of the paper. In the rest of the paper we will use $\partial_{t}$ for differentiation in the time variable. For the spatial gradient of scalars, we will use the notation $\nabla$, whereas for the full spatial derivative of vectors and tensors, we will instead use $D$. The notation $\nabla$ will also be employed for directional derivatives of the form $v \cdot \nabla=\sum_{i=1}^{3} v_{i} \frac{\partial}{\partial x_{i}}$ for scalars, vectors and tensor fields alike. Finally, by considering maps from $\mathbb{T}^{3}$ to $\mathbb{T}^{3}$ as periodic $\mathbb{R}^{3}$-valued maps, we will use the notation just described for their derivatives.

After recalling in Section 1 some preliminary notation from the paper [11], in Section 2 we give the precise definition of the maps $\left(v_{q+1}, p_{q+1}, \stackrel{\circ}{R}_{q+1}\right)$ assuming the triple $\left(v_{q}, p_{q}, \stackrel{\circ}{R}_{q}\right)$ to be known. Sections 3,4 and 5 will focus on estimating, respectively, $w_{q+1}=v_{q+1}-v_{q}, \int\left|v_{q+1}\right|^{2}(x, t) d x$ and $\stackrel{\circ}{R}_{q+1}$. These estimates are then collected in Section 6 , where Proposition 0.2 will be finally proved. In the last section we discuss a recent result [1] that adapts the present work in order to prove a weak version of Onsager's conjecture. The appendix collects several technical (and, for the most part, well-known) estimates on the different classical PDEs involved in our construction, i.e., the transport equation, the Poisson equation and the biLaplace equation.

0.4. Acknowledgements. T. B. and L. Sz. acknowledge the support of the ERC Grant Agreement No. 277993, C. dL. acknowledges the support of the SNF Grant 129812 and P. I. acknowledges the support of the NSF Graduate Research Fellowship Grant DGE-1128900.

\section{Preliminaries}

1.1. Geometric preliminaries. In this paper we denote by $\mathbb{R}^{n \times n}$, as usual, the space of $n \times n$ matrices, whereas $\mathcal{S}^{n \times n}$ and $\mathcal{S}_{0}^{n \times n}$ denote, respectively, the 
corresponding subspaces of symmetric matrices and of trace-free symmetric matrices. The $3 \times 3$ identity matrix will be denoted with Id. For definitiveness we will use the matrix operator norm $|R|:=\max _{|v|=1}|R v|$. Since we will deal with symmetric matrices, we have the identity $|R|=\max _{|v|=1}|R v \cdot v|$.

Proposition 1.1 (Beltrami flows). Let $\bar{\lambda} \geq 1$, and let $A_{k} \in \mathbb{R}^{3}$ be such that

$$
A_{k} \cdot k=0,\left|A_{k}\right|=\frac{1}{\sqrt{2}}, A_{-k}=A_{k}
$$

for $k \in \mathbb{Z}^{3}$ with $|k|=\bar{\lambda}$. Furthermore, let

$$
B_{k}=A_{k}+i \frac{k}{|k|} \times A_{k} \in \mathbb{C}^{3} .
$$

For any choice of $a_{k} \in \mathbb{C}$ with $\overline{a_{k}}=a_{-k}$, the vector field

$$
W(\xi)=\sum_{|k|=\bar{\lambda}} a_{k} B_{k} e^{i k \cdot \xi}
$$

is real-valued, divergence-free and satisfies

$$
\operatorname{div}(W \otimes W)=\nabla \frac{|W|^{2}}{2} .
$$

Furthermore,

$$
\langle W \otimes W\rangle=f_{\mathbb{T}^{3}} W \otimes W d \xi=\frac{1}{2} \sum_{|k|=\bar{\lambda}}\left|a_{k}\right|^{2}\left(\mathrm{Id}-\frac{k}{|k|} \otimes \frac{k}{|k|}\right) .
$$

The proof of (20), which is quite elementary and is included in Appendix A (see also [11]), is based on the following algebraic identity, which we state separately for future reference.

Lemma 1.2. Let $k, k^{\prime} \in \mathbb{Z}^{3}$ with $|k|=\left|k^{\prime}\right|=\bar{\lambda}$, and let $B_{k}, B_{k^{\prime}} \in \mathbb{C}^{3}$ be the associated vectors from Proposition 1.1. Then we have

$$
\left(B_{k} \otimes B_{k^{\prime}}+B_{k^{\prime}} \otimes B_{k}\right)\left(k+k^{\prime}\right)=\left(B_{k} \cdot B_{k^{\prime}}\right)\left(k+k^{\prime}\right) .
$$

Proof. The proof is a straight-forward calculation. Indeed, since $B_{k} \cdot k=$ $B_{k^{\prime}} \cdot k^{\prime}=0$, we have

$$
\begin{gathered}
\left(B_{k} \otimes B_{k^{\prime}}+B_{k^{\prime}} \otimes B_{k}\right)\left(k+k^{\prime}\right)=\left(B_{k^{\prime}} \cdot k\right) B_{k}+\left(B_{k} \cdot k^{\prime}\right) B_{k^{\prime}} \\
=-B_{k} \times\left(k^{\prime} \times B_{k^{\prime}}\right)-B_{k^{\prime}} \times\left(k \times B_{k}\right)+\left(B_{k} \cdot B_{k^{\prime}}\right)\left(k+k^{\prime}\right) \\
=i \bar{\lambda}\left(B_{k} \times B_{k^{\prime}}+B_{k^{\prime}} \times B_{k}\right)+\left(B_{k} \cdot B_{k^{\prime}}\right)\left(k+k^{\prime}\right),
\end{gathered}
$$

where the last equality follows from

$$
k \times B_{k}=-i \bar{\lambda} B_{k} \text { and } k^{\prime} \times B_{k^{\prime}}=-i \bar{\lambda} B_{k^{\prime}} .
$$

Another important ingredient is the following geometric lemma, also taken from [11]. For the reader's convenience, we give a different proof in Appendix B, following [18]. 
Lemma 1.3 (Geometric lemma). For every $N \in \mathbb{N}$, we can choose $r_{0}>0$ and $\bar{\lambda}>1$ with the following property. There exist pairwise disjoint subsets

$$
\Lambda_{j} \subset\left\{k \in \mathbb{Z}^{3}:|k|=\bar{\lambda}\right\} \quad j \in\{1, \ldots, N\}
$$

and smooth positive functions

$$
\gamma_{k}^{(j)} \in C^{\infty}\left(B_{r_{0}}(\mathrm{Id})\right) \quad j \in\{1, \ldots, N\}, k \in \Lambda_{j}
$$

such that

(a) $k \in \Lambda_{j}$ implies $-k \in \Lambda_{j}$ and $\gamma_{k}^{(j)}=\gamma_{-k}^{(j)}$;

(b) for each $R \in B_{r_{0}}(\mathrm{Id})$, we have the identity

$$
R=\frac{1}{2} \sum_{k \in \Lambda_{j}}\left(\gamma_{k}^{(j)}(R)\right)^{2}\left(\mathrm{Id}-\frac{k}{|k|} \otimes \frac{k}{|k|}\right) \quad \forall R \in B_{r_{0}}(\mathrm{Id}) .
$$

1.2. The operator $\mathcal{R}$. Following [11], we introduce the following operator in order to deal with the Reynolds stresses.

Definition 1.4. Let $v \in C^{\infty}\left(\mathbb{T}^{3}, \mathbb{R}^{3}\right)$ be a smooth vector field. We then define $\mathcal{R} v$ to be the matrix-valued periodic function

$$
\mathcal{R} v:=\frac{1}{4}\left(D \mathcal{P} u+(D \mathcal{P} u)^{T}\right)+\frac{3}{4}\left(D u+(D u)^{T}\right)-\frac{1}{2}(\operatorname{div} u) \operatorname{Id},
$$

where $u \in C^{\infty}\left(\mathbb{T}^{3}, \mathbb{R}^{3}\right)$ is the solution of

$$
\Delta u=v-f_{\mathbb{T}^{3}} v \text { in } \mathbb{T}^{3}
$$

with $f_{\mathbb{T}^{3}} u=0$ and $\mathcal{P}$ is the Leray projection onto divergence-free fields with zero average.

Lemma $1.5\left(\mathcal{R}=\operatorname{div}^{-1}\right)$. For any $v \in C^{\infty}\left(\mathbb{T}^{3}, \mathbb{R}^{3}\right)$, we have

(a) $\mathcal{R} v(x)$ is a symmetric trace-free matrix for each $x \in \mathbb{T}^{3}$,

(b) $\operatorname{div} \mathcal{R} v=v-f_{\mathbb{T}^{3}} v$.

The proof is elementary; we include it for the reader's convenience.

Proof. It is obvious by inspection that $\mathcal{R} v$ is symmetric. Since $\operatorname{div} \mathcal{P} v=0$, for the trace we obtain

Similarly, we have

$$
\operatorname{tr}(\mathcal{R} v)=\frac{3}{4}(2 \operatorname{div} u)-\frac{3}{2} \operatorname{div} u=0 .
$$

$$
\operatorname{div}(\mathcal{R} v)=\frac{1}{4} \Delta(\mathcal{P} u)+\frac{3}{4}(\nabla \operatorname{div} u+\Delta u)-\frac{1}{2} \nabla \operatorname{div} u .
$$

On the other hand, recall that $\mathcal{P} u=u-\nabla \phi-f u=u-\nabla \phi$, where $\Delta \phi=\operatorname{div} u$. Therefore $\Delta(\mathcal{P} u)=\Delta u-\nabla \operatorname{div} u$. Plugging this identity into (24), we obtain

$$
\operatorname{div}(\mathcal{R} v)=\Delta u
$$

and since $u$ solves $\Delta u=v-f v$, (b) follows readily. 


\section{The inductive step}

In this section we specify the inductive procedure that allows us to construct $\left(v_{q+1}, p_{q+1}, \stackrel{\circ}{R}_{q+1}\right)$ from $\left(v_{q}, p_{q}, \stackrel{\circ}{R}_{q}\right)$. Note that the choice of the sequences $\left\{\delta_{q}\right\}_{q \in \mathbb{N}}$ and $\left\{\lambda_{q}\right\}_{q \in \mathbb{N}}$ specified in Proposition 0.2 implies that, for a sufficiently large $a>1$, depending only on $b>1$ and $c>5 / 2$, we have

$$
\sum_{j \leq q} \delta_{j} \lambda_{j} \leq 2 \delta_{q} \lambda_{q}, \quad 1 \leq \sum_{j \leq q} \delta_{j}^{1 / 2} \lambda_{j} \leq 2 \delta_{q}^{1 / 2} \lambda_{q}, \quad \sum_{j} \delta_{j} \leq \sum_{j} \delta_{j}^{1 / 2} \leq 2 .
$$

Since we are concerned with a single step in the iteration, with a slight abuse of notation we will write $(v, p, \stackrel{\circ}{R})$ instead of $\left(v_{q}, p_{q}, \stackrel{\circ}{R}_{q}\right)$ and $\left(v_{1}, p_{1}, \stackrel{\circ}{R}_{1}\right)$ instead of $\left(v_{q+1}, p_{q+1}, R_{q+1}\right)$. Our inductive hypothesis then implies the following set of estimates:

$$
\begin{array}{ll}
\|v\|_{0} \leq 2 M, & \|v\|_{1} \leq 2 M \delta_{q}^{1 / 2} \lambda_{q}, \\
\|\stackrel{\circ}{R}\|_{0} \leq \eta \delta_{q+1}, & \|\stackrel{\circ}{R}\|_{1} \leq M \delta_{q+1} \lambda_{q} \\
\|p\|_{0} \leq 2 M^{2}, & \|p\|_{1} \leq 2 M^{2} \delta_{q} \lambda_{q}
\end{array}
$$

and

$$
\left\|\left(\partial_{t}+v \cdot \nabla\right) \stackrel{\circ}{R}\right\|_{0} \leq M \delta_{q+1} \delta_{q}^{1 / 2} \lambda_{q} .
$$

The new velocity $v_{1}$ will be defined as a sum

$$
v_{1}:=v+w_{o}+w_{c}
$$

where $w_{o}$ is the principal perturbation and $w_{c}$ is a corrector. The "principal part" of the perturbation $w$ will be a sum of Beltrami flows

$$
w_{o}(t, x):=\sum_{|k|=\lambda_{0}} a_{k}(t, x) \phi_{k}(t, x) B_{k} e^{i \lambda_{q+1} k \cdot x},
$$

where $B_{k} e^{i \lambda_{q+1} k \cdot x}$ is a single Beltrami mode at frequency $\lambda_{q+1}$, with phase shift $\phi_{k}=\phi_{k}(t, x)$ (i.e., $\left|\phi_{k}\right|=1$ ) and amplitude $a_{k}=a_{k}(t, x)$. In the following subsections we will define $a_{k}$ and $\phi_{k}$.

2.1. Space regularization of $v$ and $R$. We fix a symmetric nonnegative convolution kernel $\psi \in C_{c}^{\infty}\left(\mathbb{R}^{3}\right)$ and a small parameter $\ell$ (whose choice will be specified later). Define $v_{\ell}:=v * \psi_{\ell}$ and $\stackrel{\circ}{R}_{\ell}:=\stackrel{\circ}{R} * \psi_{\ell}$, where the convolution is in the $x$ variable only. Standard estimates on regularizations by convolution lead to the following:

$$
\begin{aligned}
& \left\|v-v_{\ell}\right\|_{0} \leq C M \delta_{q}^{1 / 2} \lambda_{q} \ell, \\
& \left\|\stackrel{\circ}{R}-\stackrel{\circ}{R}_{\ell}\right\|_{0} \leq C M \delta_{q+1} \lambda_{q} \ell,
\end{aligned}
$$


and for any $N \geq 1$, there exists a constant $C=C(N)$ so that

$$
\begin{aligned}
& \left\|v_{\ell}\right\|_{N} \leq C M \delta_{q}^{1 / 2} \lambda_{q} \ell^{1-N}, \\
& \left\|\stackrel{\circ}{R}_{\ell}\right\|_{N} \leq C M \delta_{q+1} \lambda_{q} \ell^{1-N} .
\end{aligned}
$$

2.2. Time discretization and transport for the Reynolds stress. Next, we fix a smooth cutoff function $\chi \in C_{c}^{\infty}\left(\left(-\frac{3}{4}, \frac{3}{4}\right)\right)$ such that

$$
\sum_{l \in \mathbb{Z}} \chi^{2}(x-l)=1
$$

and a large parameter $\mu \in \mathbb{N} \backslash\{0\}$, whose choice will be specified later.

For any $l \in[0, \mu]$, we define

$$
\rho_{l}:=\frac{1}{3(2 \pi)^{3}}\left(e\left(l \mu^{-1}\right)\left(1-\delta_{q+2}\right)-\int_{\mathbb{T}^{3}}|v|^{2}\left(x, l \mu^{-1}\right) d x\right) .
$$

Note that (12) implies

$$
\frac{1}{3(2 \pi)^{3}} e\left(l \mu^{-1}\right)\left(\frac{3}{4} \delta_{q+1}-\delta_{q+2}\right) \leq \rho_{l} \leq \frac{1}{3(2 \pi)^{3}} e\left(l \mu^{-1}\right)\left(\frac{5}{4} \delta_{q+1}-\delta_{q+2}\right) .
$$

Recalling that $b$ and $c$ are fixed, whereas $a$ is chosen large, since $\delta_{q}=a^{-b^{q}}$, we will assume $\delta_{q+2} \leq \frac{1}{2} \delta_{q+1}$ so that we obtain

$$
C_{0}^{-1}(\min e) \delta_{q+1} \leq \rho_{l} \leq C_{0}(\max e) \delta_{q+1},
$$

where $C_{0}$ is an absolute constant.

Finally, define $R_{\ell, l}$ to be the unique solution to the transport equation

$$
\left\{\begin{array}{l}
\partial_{t} \stackrel{\circ}{R}_{\ell, l}+v_{\ell} \cdot \nabla \stackrel{\circ}{R}_{\ell, l}=0, \\
\stackrel{\circ}{R}_{\ell, l}\left(x, \frac{l}{\mu}\right)=\stackrel{\circ}{R}_{\ell}\left(x, \frac{l}{\mu}\right),
\end{array}\right.
$$

and set

$$
R_{\ell, l}(x, t):=\rho_{l} \mathrm{Id}-\stackrel{\circ}{R}_{\ell, l}(x, t) .
$$

2.3. The maps $v_{1}, w, w_{o}$ and $w_{c}$. We next consider $v_{\ell}$ as a $2 \pi$-periodic function on $\mathbb{R}^{3} \times[0,1]$ and, for every $l \in[0, \mu]$, we let $\Phi_{l}: \mathbb{R}^{3} \times[0,1] \rightarrow \mathbb{R}^{3}$ be the solution of

$$
\left\{\begin{array}{l}
\partial_{t} \Phi_{l}+v_{\ell} \cdot \nabla \Phi_{l}=0, \\
\Phi_{l}\left(x, l \mu^{-1}\right)=x
\end{array}\right.
$$

Observe that $\Phi_{l}(\cdot, t)$ is the inverse of the flow of the periodic vector-field $v_{\ell}$, starting at time $t=l \mu^{-1}$ as the identity. Thus, if $y \in(2 \pi \mathbb{Z})^{3}$, then $\Phi_{l}(x, t)-$ $\Phi_{l}(x+y, t) \in(2 \pi \mathbb{Z})^{3}: \Phi_{l}(\cdot, t)$ can be thought as a diffeomorphism of $\mathbb{T}^{3}$ onto itself and, for every $k \in \mathbb{Z}^{3}$, the map $\mathbb{T}^{3} \times[0,1] \ni(x, t) \rightarrow e^{i \lambda_{q+1} k \cdot \Phi_{l}(x, t)}$ is well defined. 
We next apply Lemma 1.3 with $N=2$, denoting by $\Lambda^{e}$ and $\Lambda^{o}$ the corresponding families of frequencies in $\mathbb{Z}^{3}$, and we set $\Lambda:=\Lambda^{o}+\Lambda^{e}$. For each $k \in \Lambda$ and each $l \in \mathbb{Z} \cap[0, \mu]$, we then set

$$
\begin{aligned}
\chi_{l}(t) & :=\chi(\mu(t-l)), \\
a_{k l}(x, t) & :=\sqrt{\rho_{l}} \gamma_{k}\left(\frac{R_{\ell, l}(x, t)}{\rho_{l}}\right), \\
w_{k l}(x, t) & :=a_{k l}(x, t) B_{k} e^{i \lambda_{q+1} k \cdot \Phi_{l}(x, t)} .
\end{aligned}
$$

The "principal part" of the perturbation $w$ consists of the map

$$
w_{o}(x, t):=\sum_{l \text { odd }, k \in \Lambda^{o}} \chi_{l}(t) w_{k l}(x, t)+\sum_{l \text { even }, k \in \Lambda^{e}} \chi_{l}(t) w_{k l}(x, t) .
$$

From now on, in order to make our notation simpler, we agree that the pairs of indices $(k, l) \in \Lambda \times[0, \mu]$ that enter in our summations always satisfy the following condition: $k \in \Lambda^{e}$ when $l$ is even and $k \in \Lambda^{o}$ when $l$ is odd.

It will be useful to introduce the "phase"

$$
\phi_{k l}(x, t)=e^{i \lambda_{q+1} k \cdot\left[\Phi_{l}(x, t)-x\right]},
$$

with which we obviously have

$$
\phi_{k l} \cdot e^{i \lambda_{q+1} k \cdot x}=e^{i \lambda_{q+1} k \cdot \Phi_{l}} .
$$

Since $R_{\ell, l}$ and $\Phi_{l}$ are defined as solutions of the transport equations (35) and (37), we have

$$
\left(\partial_{t}+v_{\ell} \cdot \nabla\right) a_{k l}=0 \quad \text { and } \quad\left(\partial_{t}+v_{\ell} \cdot \nabla\right) e^{i \lambda_{q+1} k \cdot \Phi_{l}(x, t)}=0,
$$

and hence also

$$
\left(\partial_{t}+v_{\ell} \cdot \nabla\right) w_{k l}=0 .
$$

The corrector $w_{c}$ is then defined in such a way that $w:=w_{o}+w_{c}$ is divergence free:

$$
\begin{aligned}
w_{c} & :=\sum_{k l} \frac{\chi_{l}}{\lambda_{q+1}} \operatorname{curl}\left(i a_{k l} \phi_{k l} \frac{k \times B_{k}}{|k|^{2}}\right) e^{i \lambda_{q+1} k \cdot x} \\
& =\sum_{k l} \chi_{l}\left(\frac{i}{\lambda_{q+1}} \nabla a_{k l}-a_{k l}\left(D \Phi_{l}-\mathrm{Id}\right) k\right) \times \frac{k \times B_{k}}{|k|^{2}} e^{i \lambda_{q+1} k \cdot \Phi_{l}} .
\end{aligned}
$$

Remark 1. To see that $w=w_{o}+w_{c}$ is divergence-free, just note that since $k \cdot B_{k}=0$, we have $k \times\left(k \times B_{k}\right)=-|k|^{2} B_{k}$ and hence $w$ can be written as

$$
w=\frac{1}{\lambda_{q+1}} \sum_{(k, l)} \chi_{l} \operatorname{curl}\left(i a_{k l} \phi_{k l} \frac{k \times B_{k}}{|k|^{2}} e^{i \lambda_{q+1} k \cdot x}\right) .
$$


For future reference it is useful to introduce the notation

$$
L_{k l}:=a_{k l} B_{k}+\left(\frac{i}{\lambda_{q+1}} \nabla a_{k l}-a_{k l}\left(D \Phi_{l}-\mathrm{Id}\right) k\right) \times \frac{k \times B_{k}}{|k|^{2}},
$$

so that the perturbation $w$ can be written as

$$
w=\sum_{k l} \chi_{l} L_{k l} e^{i \lambda_{q+1} k \cdot \Phi_{l}} .
$$

Moreover, we will frequently deal with the transport derivative with respect to the regularized flow $v_{\ell}$ of various expressions, and we will henceforth use the notation

$$
D_{t}:=\partial_{t}+v_{\ell} \cdot \nabla
$$

2.4. Determination of the constants $\eta$ and $M$. In order to determine $\eta$, first of all recall from Lemma 1.3 that the functions $a_{k l}$ are well defined provided

$$
\left|\frac{R_{\ell, l}}{\rho_{l}}-\mathrm{Id}\right| \leq r_{0},
$$

where $r_{0}$ is the constant of Lemma 1.3. Recalling the definition of $R_{\ell, l}$, we easily deduce from the maximum principle for transport equations (cf. (132) in Proposition D.1) that $\left\|\stackrel{\circ}{R}_{\ell, l}\right\|_{0} \leq\|\stackrel{\circ}{R}\|_{0}$. Hence, from (10) and (34), we obtain

$$
\left|\frac{R_{\ell, l}}{\rho_{l}}-\mathrm{Id}\right| \leq C_{0} \frac{\eta}{\min e},
$$

and thus we will require that

$$
C_{0} \frac{\eta}{\min e} \leq \frac{r_{0}}{4}
$$

The constant $M$ in turn is determined by comparing the estimate (6) for $q+1$ with the definition of the principal perturbation $w_{o}$ in (41). Indeed, using (38)-(41) and (34) we have $\left\|w_{o}\right\|_{0} \leq C_{0}|\Lambda|(\max e) \delta_{q+1}^{1 / 2}$. We therefore set

$$
M=2 C_{0}|\Lambda|(\max e)
$$

so that

$$
\left\|w_{o}\right\|_{0} \leq \frac{M}{2} \delta_{q+1}^{1 / 2}
$$

2.5. The pressure $p_{1}$ and the Reynolds stress $\stackrel{\circ}{R}_{1}$. We set

$$
\stackrel{\circ}{R}_{1}=R^{0}+R^{1}+R^{2}+R^{3}+R^{4}+R^{5},
$$


where

$$
\begin{aligned}
& R^{0}=\mathcal{R}\left(\partial_{t} w+v_{\ell} \cdot \nabla w+w \cdot \nabla v_{\ell}\right), \\
& R^{1}=\mathcal{R} \operatorname{div}\left(w_{o} \otimes w_{o}-\sum_{l} \chi_{l}^{2} R_{\ell, l}-\frac{\left|w_{o}\right|^{2}}{2} \mathrm{Id}\right), \\
& R^{2}=w_{o} \otimes w_{c}+w_{c} \otimes w_{o}+w_{c} \otimes w_{c}-\frac{\left|w_{c}\right|^{2}+2\left\langle w_{o}, w_{c}\right\rangle}{3} \mathrm{Id}, \\
& R^{3}=w \otimes\left(v-v_{\ell}\right)+\left(v-v_{\ell}\right) \otimes w-\frac{2\left\langle\left(v-v_{\ell}\right), w\right\rangle}{3} \mathrm{Id}, \\
& R^{4}=\stackrel{\circ}{R}-\stackrel{\circ}{R}_{\ell} \\
& R^{5}=\sum_{l} \chi_{l}^{2}\left(\stackrel{\circ}{R}_{\ell}-\stackrel{\circ}{R}_{l, \ell}\right) .
\end{aligned}
$$

Observe that $\stackrel{\circ}{R}_{1}$ is indeed a traceless symmetric tensor. The corresponding form of the new pressure will then be

$$
p_{1}=p-\frac{\left|w_{o}\right|^{2}}{2}-\frac{1}{3}\left|w_{c}\right|^{2}-\frac{2}{3}\left\langle w_{o}, w_{c}\right\rangle-\frac{2}{3}\left\langle v-v_{\ell}, w\right\rangle .
$$

Recalling (36) we see that $\sum_{l} \chi_{l}^{2} \operatorname{tr} R_{\ell, l}$ is a function of time only. Since also $\sum_{l} \chi_{l}^{2}=1$, it is then straightforward to check that

$$
\begin{aligned}
\operatorname{div} \stackrel{\circ}{R}_{1}-\nabla p_{1} & =\partial_{t} w+\operatorname{div}(v \otimes w+w \otimes v+w \otimes w)+\operatorname{div} \stackrel{\circ}{R}-\nabla p \\
& =\partial_{t} w+\operatorname{div}(v \otimes w+w \otimes v+w \otimes w)+\partial_{t} v+\operatorname{div}(v \otimes v) \\
& =\partial_{t} v_{1}+\operatorname{div}\left(v_{1} \otimes v_{1}\right) .
\end{aligned}
$$

The following lemma will play a key role.

\section{LEMMA 2.1. The following identity holds:}

$$
w_{o} \otimes w_{o}=\sum_{l} \chi_{l}^{2} R_{\ell, l}+\sum_{(k, l),\left(k^{\prime}, l^{\prime}\right), k \neq-k^{\prime}} \chi_{l} \chi_{l^{\prime}} w_{k l} \otimes w_{k^{\prime} l^{\prime}} .
$$

Proof. Recall that the pairs $(k, l),\left(k^{\prime}, l^{\prime}\right)$ are chosen so that $k \neq-k^{\prime}$ if $l$ is even and $l^{\prime}$ is odd. Moreover, $\chi_{l} \chi_{l^{\prime}}=0$ if $l$ and $l^{\prime}$ are distinct and have the same parity. Hence the claim follows immediately from our choice of $a_{k l}$ in (39) and Proposition 1.1 and Lemma 1.3 (cf. [11, Prop. 6.1(ii)]).

2.6. Conditions on the parameters - hierarchy of length-scales. In the next couple of sections we will need to estimate various expressions involving $v_{\ell}$ and $w$. To simplify the formulas that we arrive at, from now on we will assume the following conditions on $\mu, \lambda_{q+1} \geq 1$ and $\ell \leq 1$ :

$$
\frac{\delta_{q}^{1 / 2} \lambda_{q} \ell}{\delta_{q+1}^{1 / 2}} \leq 1, \quad \frac{\delta_{q}^{1 / 2} \lambda_{q}}{\mu}+\frac{1}{\ell \lambda_{q+1}} \leq \lambda_{q+1}^{-\beta} \quad \text { and } \quad \frac{1}{\lambda_{q+1}} \leq \frac{\delta_{q+1}^{1 / 2}}{\mu} .
$$


These conditions imply the following orderings of length scales, which will be used to simplify the estimates in Section 3:

$$
\frac{1}{\delta_{q+1}^{1 / 2} \lambda_{q+1}} \leq \frac{1}{\mu} \leq \frac{1}{\delta_{q}^{1 / 2} \lambda_{q}} \quad \text { and } \quad \frac{1}{\lambda_{q+1}} \leq \ell \leq \frac{1}{\lambda_{q}} .
$$

One can think of these chains of inequalities as an ordering of various length scales involved in the definition of $v_{1}$.

Remark 2. The most relevant and restrictive condition is $\delta_{q}^{1 / 2} \lambda_{q} \leq \mu$. Indeed, this condition can be thought of as a kind of CFL condition (cf. [5]), restricting the coarse-grained flow to times of the order of $\|\nabla v\|_{0}^{-1}$; cf. Lemma 3.1 and in particular, (62) below. Assuming only this condition on the parameters, essentially all the arguments for estimating the various terms would still follow through. The remaining inequalities are only used to simplify the many estimates needed in the rest of the paper, which otherwise would have a much more complicated dependence upon the various parameters.

\section{Estimates on the perturbation}

Lemma 3.1. Assume (59) holds. For $t$ in the range $|\mu t-l|<1$, we have

$$
\begin{array}{ll}
\left\|D \Phi_{l}\right\|_{0} \leq C \\
\left\|D \Phi_{l}-\mathrm{Id}\right\|_{0} \leq C \frac{\delta_{q}^{1 / 2} \lambda_{q}}{\mu}, & \\
\left\|D \Phi_{l}\right\|_{N} \leq C \frac{\delta_{q}^{1 / 2} \lambda_{q}}{\mu \ell^{N}}, & N \geq 1 .
\end{array}
$$

Moreover,

$$
\begin{aligned}
& \left\|a_{k l}\right\|_{0}+\left\|L_{k l}\right\|_{0} \leq C \delta_{q+1}^{1 / 2}, \\
& \left\|a_{k l}\right\|_{N} \leq C \delta_{q+1}^{1 / 2} \lambda_{q} \ell^{1-N}, \quad N \geq 1 \\
& \left\|L_{k l}\right\|_{N} \leq C \delta_{q+1}^{1 / 2} \ell^{-N}, \quad N \geq 1 \\
& \left\|\phi_{k l}\right\|_{N} \leq C \lambda_{q+1} \frac{\delta_{q}^{1 / 2} \lambda_{q}}{\mu \ell^{N-1}}+C\left(\frac{\delta_{q}^{1 / 2} \lambda_{q} \lambda_{q+1}}{\mu}\right)^{N} \\
& \leq C \lambda_{q+1}^{N(1-\beta)} \\
& N \geq 1
\end{aligned}
$$

Consequently, for any $N \geq 0$,

$$
\begin{aligned}
\left\|w_{c}\right\|_{N} & \leq C \delta_{q+1}^{1 / 2} \frac{\delta_{q}^{1 / 2} \lambda_{q}}{\mu} \lambda_{q+1}^{N}, \\
\left\|w_{o}\right\|_{1} \leq \frac{M}{2} \delta_{q+1}^{1 / 2} \lambda_{q+1}+C \delta_{q+1}^{1 / 2} \lambda_{q+1}^{1-\beta}, & \\
\left\|w_{o}\right\|_{N} \leq C \delta_{q+1}^{1 / 2} \lambda_{q+1}^{N}, & N \geq 2,
\end{aligned}
$$


where the constants in (61)-(62) depend only on $M$, the constant in (63) depends on $M$ and $N$, the constants in (64) and (69) depend on $M$ and $e$ and the remaining constants depend on $M, e$ and $N$.

Proof. The estimates (61) and (62) are direct consequences of (135) in Proposition D.1, together with (60), whereas (136) in Proposition D.1 combined with the convolution estimate (32) implies (63).

Next, (33) together with (132), (133) and (134) in Proposition D.1 and (60) leads to

$$
\begin{aligned}
& \left\|R_{\ell, l}\right\|_{0} \leq C \delta_{q+1}, \\
& \left\|R_{\ell, l}\right\|_{N} \leq C \delta_{q+1} \lambda_{q} \ell^{1-N}, \quad N \geq 1 .
\end{aligned}
$$

The estimate (64) is now a consequence of (71), (62) and (34), whereas by (129) we obtain

$$
\left\|a_{k l}\right\|_{N} \leq C \delta_{q+1}^{-1 / 2}\left\|R_{\ell, l}\right\|_{N} \leq C \delta_{q+1}^{1 / 2} \lambda_{q} \ell^{1-N} \leq C \delta_{q+1}^{1 / 2} \ell^{-N} .
$$

Similarly, we deduce (66) from

$$
\begin{aligned}
\left\|L_{k l}\right\|_{N} \leq & C\left\|a_{k l}\right\|_{N}+C \lambda_{q+1}^{-1}\left\|a_{k l}\right\|_{N+1} \\
& +C\left(\left\|a_{k l}\right\|_{N}\left\|D \Phi_{l}-\mathrm{Id}\right\|_{0}+\left\|a_{k l}\right\|_{0}\left\|D \Phi_{l}\right\|_{N}\right),
\end{aligned}
$$

once again using (60).

In order to prove (67) we apply (130) with $m=N$ to conclude

$$
\left\|\phi_{k l}\right\|_{N} \leq C \lambda_{q+1}\left\|D \Phi_{l}\right\|_{N-1}+\lambda_{q+1}^{N}\left\|D \Phi_{l}-\operatorname{Id}\right\|_{0}^{N},
$$

from which (67) follows using (62), (63) and (59).

Using the formula (45) together with (62), (63), (64) and (66) we conclude

$$
\left\|w_{c}\right\|_{0} \leq \frac{C}{\lambda_{q+1}}\left\|a_{k l}\right\|_{1}+C\left\|a_{k l}\right\|_{0}\left\|D \Phi_{l}-\mathrm{Id}\right\|_{0} \leq C \frac{\delta_{q}^{1 / 2} \lambda_{q}}{\mu}
$$

and, for $N \geq 1$,

$$
\begin{aligned}
\left\|w_{c}\right\|_{N} \leq & C \sum_{k l} \chi_{l}\left(\frac{1}{\lambda_{q+1}}\left\|a_{k l}\right\|_{N+1}+\left\|a_{k l}\right\|_{0}\left\|D \Phi_{l}\right\|_{N}+\left\|a_{k l}\right\|_{N}\left\|D \Phi_{l}-\mathrm{Id}\right\|_{0}\right) \\
& +C\left\|w_{c}\right\|_{0} \sum_{l} \chi_{l}\left(\lambda_{q+1}^{N}\left\|D \Phi_{l}\right\|_{0}^{N}+\lambda_{q+1}\left\|D \Phi_{l}\right\|_{N-1}\right) \\
& \stackrel{(60)}{\leq} C \delta_{q+1}^{1 / 2} \lambda_{q+1}^{N}\left(\frac{\lambda_{q}}{\lambda_{q+1}}+\frac{\delta_{q}^{1 / 2} \lambda_{q}}{\mu}\right) \leq C \frac{\delta_{q}^{1 / 2} \lambda_{q}}{\mu} \lambda_{q+1}^{N} .
\end{aligned}
$$

This proves (68). The estimates for $w_{o}$ follow analogously, using in addition the choice of $M$ and (50). 
Lemma 3.2. Recall that $D_{t}=\partial_{t}+v_{\ell} \cdot \nabla$. Under the assumptions of Lemma 3.1, we have

$$
\begin{aligned}
\left\|D_{t} v_{\ell}\right\|_{N} & \leq C \delta_{q} \lambda_{q} \ell^{-N}, \\
\left\|D_{t} L_{k l}\right\|_{N} & \leq C \delta_{q+1}^{1 / 2} \delta_{q}^{1 / 2} \lambda_{q} \ell^{-N}, \\
\left\|D_{t}^{2} L_{k l}\right\|_{N} & \leq C \delta_{q+1}^{1 / 2} \delta_{q} \lambda_{q} \ell^{-N-1}, \\
\left\|D_{t} w_{c}\right\|_{N} & \leq C \delta_{q+1}^{1 / 2} \delta_{q}^{1 / 2} \lambda_{q} \lambda_{q+1}^{N}, \\
\left\|D_{t} w_{o}\right\|_{N} & \leq C \delta_{q+1}^{1 / 2} \mu \lambda_{q+1}^{N} .
\end{aligned}
$$

Proof. Estimate on $D_{t} v_{\ell}$. Note that $v_{\ell}$ satisfies the inhomogeneous transport equation

$$
\partial_{t} v_{\ell}+v_{\ell} \cdot \nabla v_{\ell}=-\nabla p * \psi_{\ell}+\operatorname{div}\left(\stackrel{\circ}{R}_{\ell}-(v \otimes v) * \psi_{\ell}+v_{\ell} \otimes v_{\ell}\right) .
$$

By hypothesis, $\left\|\nabla p * \psi_{\ell}\right\|_{N} \leq C\|p\|_{1} \ell^{-N} \leq C \delta_{q} \lambda_{q} \ell^{-N}$ and analogously $\| \operatorname{div} \stackrel{\circ}{R} *$ $\psi_{\ell} \| \leq C \delta_{q+1} \lambda_{q} \ell^{-N}$. On the other hand, by Proposition E.1,

$$
\left\|\operatorname{div}\left((v \otimes v) * \psi_{\ell}-v_{\ell} \otimes v_{\ell}\right)\right\|_{N} \leq C \ell^{1-N}\|v\|_{1}^{2} \leq C \ell^{1-N} \delta_{q} \lambda_{q}^{2} .
$$

Thus (74) follows from (60).

Estimates on $L_{k l}$. Recall that $L_{k l}$ is defined as

$$
L_{k l}:=a_{k l} B_{k}+\left(\frac{i}{\lambda_{q+1}} \nabla a_{k l}-a_{k l}\left(D \Phi_{l}-\mathrm{Id}\right) k\right) \times \frac{k \times B_{k}}{|k|^{2}} .
$$

Using that

$$
\begin{aligned}
D_{t} a_{k l} & =0, \quad D_{t} \Phi_{l}=0, \\
D_{t} \nabla a_{k l} & =-D v_{\ell}^{T} \nabla a_{k l}, \quad D_{t} D \Phi_{l}=-D \Phi_{l} D v_{\ell},
\end{aligned}
$$

we obtain

$$
D_{t} L_{k l}=\left(-\frac{i}{\lambda_{q+1}} D v_{\ell}^{T} \nabla a_{k l}+a_{k l} D \Phi_{l} D v_{\ell} k\right) \times \frac{k \times B_{k}}{|k|^{2}} .
$$

Consequently, for times $|t-l|<\mu^{-1}$ and $N \geq 0$, we have

$$
\begin{aligned}
\left\|D_{t} L_{k l}\right\|_{N} & \leq C \delta_{q+1}^{1 / 2} \delta_{q}^{1 / 2} \lambda_{q} \ell^{-N}\left(\frac{\lambda_{q}}{\lambda_{q+1}}+\lambda_{q} \ell+\frac{\delta_{q}^{1 / 2} \lambda_{q}}{\mu}+1\right) \\
& \leq C \delta_{q+1}^{1 / 2} \delta_{q}^{1 / 2} \lambda_{q} \ell^{-N}
\end{aligned}
$$

where we have used (127), Lemma 3.1 and (60). Taking one more derivative and using (79) again, we obtain

$$
\begin{aligned}
D_{t}^{2} L_{k l}=( & -\frac{i}{\lambda_{q+1}}\left(D_{t} D v_{\ell}\right)^{T} \nabla a_{k l}+\frac{i}{\lambda_{q+1}} D v_{\ell}^{T} D v_{\ell}^{T} \nabla a_{k l} \\
& \left.-a_{k l} D \Phi_{l} D v_{\ell} D v_{\ell} k+a_{k l} D \Phi_{l} D_{t} D v_{\ell} k\right) \times \frac{k \times B_{k}}{|k|^{2}} .
\end{aligned}
$$


Note that $D_{t} D v_{\ell}=D D_{t} v_{\ell}-D v_{\ell} D v_{\ell}$, so that

$$
\begin{aligned}
\left\|D_{t} D v_{\ell}\right\|_{N} & \leq\left\|D_{t} v_{\ell}\right\|_{N+1}+\left\|D v_{\ell}\right\|_{N}\left\|D v_{\ell}\right\|_{0} \\
& \leq C \delta_{q} \lambda_{q} \ell^{-N-1}\left(1+\lambda_{q} \ell\right) \leq C \delta_{q} \lambda_{q} \ell^{-N-1} .
\end{aligned}
$$

It then follows from the product rule (127) and (60) that

$$
\begin{aligned}
\left\|D_{t}^{2} L_{k l}\right\|_{N} & \leq C \delta_{q+1}^{1 / 2} \delta_{q} \lambda_{q} \ell^{-N-1}\left(\frac{\lambda_{q}}{\lambda_{q+1}}+\frac{\lambda_{q}^{2} \ell}{\lambda_{q+1}}+\lambda_{q} \ell+\left(\lambda_{q} \ell\right)^{2}+\frac{\delta_{q}^{1 / 2} \lambda_{q}}{\mu}+1\right) \\
& \leq C \delta_{q+1}^{1 / 2} \delta_{q} \lambda_{q} \ell^{-N-1}
\end{aligned}
$$

Estimates on $w_{c}$. Observe that $w_{c}=\sum \chi_{l}\left(L_{k l}-a_{k l} B_{k}\right) e^{i \lambda_{q+1} k \cdot \Phi_{l}}$ (see (45) and (47)). Differentiating this identity, we then conclude

$$
\begin{aligned}
D_{t} w_{c}= & \sum_{k l} \chi_{l}\left(D_{t} L_{k l}\right) e^{i \lambda_{q+1} k \cdot \Phi_{l}}+\left(\partial_{t} \chi_{l}\right)\left(L_{k l}-a_{k l} B_{k}\right) e^{i \lambda_{q+1} k \cdot \Phi_{l}} \\
= & \sum_{k l} \chi_{l}\left(D_{t} L_{k l}\right) \phi_{k l} e^{i \lambda_{q+1} k \cdot x}+ \\
& +\sum_{k l}\left(\partial_{t} \chi_{l}\right)\left(\frac{i \nabla a_{k l}}{\lambda_{q+1}}-a_{k l}\left(D \Phi_{l}-\mathrm{Id}\right) k\right) \times \frac{k \times B_{k}}{|k|^{2}} \phi_{k l} e^{i \lambda_{q+1} k \cdot x} .
\end{aligned}
$$

Hence we obtain (77) as a consequence of Lemma 3.1 and (75).

Estimates on $w_{o}$. Using (79), we have

$$
D_{t} w_{o}=\sum_{k, l} \chi_{l}^{\prime} a_{k l} \phi_{k l} e^{i \lambda_{q+1} k \cdot x} .
$$

Therefore (78) follows immediately from Lemma 3.1.

\section{Estimates on the energy}

LEMma 4.1 (Estimate on the energy).

$$
\left.\left|e(t)\left(1-\delta_{q+2}\right)-\int_{\mathbb{T}^{3}}\right| v_{1}\right|^{2} d x \mid \leq \frac{1}{\mu}+C \frac{\delta_{q+1} \delta_{q}^{1 / 2} \lambda_{q}}{\mu}+C \frac{\delta_{q+1}^{1 / 2} \delta_{q}^{1 / 2} \lambda_{q}}{\lambda_{q+1}} .
$$

Proof. Define

$$
\bar{e}(t):=3(2 \pi)^{3} \sum_{l} \chi_{l}^{2}(t) \rho_{l} .
$$

Using Lemma 2.1, we then have

$$
\begin{aligned}
\left|w_{o}\right|^{2} & =\sum_{l} \chi_{l}^{2} \operatorname{tr} R_{\ell, l}+\sum_{(k, l),\left(k^{\prime}, l^{\prime}\right), k \neq-k^{\prime}} \chi_{l} \chi_{l^{\prime}} w_{k l} \cdot w_{k, l^{\prime}} \\
& =(2 \pi)^{-3} \bar{e}+\sum_{(k, l),\left(k^{\prime}, l^{\prime}\right), k \neq-k^{\prime}} \chi_{l} \chi_{k^{\prime}} a_{k l} a_{k^{\prime} l^{\prime}} \phi_{k l} \phi_{k^{\prime} l^{\prime}} e^{i \lambda_{q+1}\left(k+k^{\prime}\right) \cdot x} .
\end{aligned}
$$


Observe that $\bar{e}$ is a function of $t$ only and that, since $\left(k+k^{\prime}\right) \neq 0$ in the sum above, we can apply Proposition G.1(i) with $m=1$. From Lemma 3.1 we then deduce

$$
\left.\left|\int_{\mathbb{T}^{3}}\right| w_{o}\right|^{2} d x-\bar{e}(t) \mid \leq C \frac{\delta_{q+1} \delta_{q}^{1 / 2} \lambda_{q}}{\mu}+C \frac{\delta_{q+1} \lambda_{q}}{\lambda_{q+1}} .
$$

Next we recall (46), integrate by parts and use (64) and (67) to reach

$$
\left|\int_{\mathbb{T}^{3}} v \cdot w d x\right| \leq C \frac{\delta_{q+1}^{1 / 2} \delta_{q}^{1 / 2} \lambda_{q}}{\lambda_{q+1}} .
$$

Note also that by (68), we have

$$
\int_{\mathbb{T}^{3}}\left|w_{c}\right|^{2}+\left|w_{c} w_{o}\right| d x \leq C \frac{\delta_{q+1} \delta_{q}^{1 / 2} \lambda_{q}}{\mu} .
$$

Summarizing, so far we have achieved

$$
\begin{aligned}
& \left.\left|\int_{\mathbb{T}^{3}}\right| v_{1}\right|^{2} d x-\left(\bar{e}(t)+\int_{\mathbb{T}^{3}}|v|^{2} d x\right)|\stackrel{(83)}{\leq}| \int_{\mathbb{T}^{3}}|w|^{2} d x-\bar{e}(t) \mid+C \frac{\delta_{q+1}^{1 / 2} \delta_{q}^{1 / 2} \lambda_{q}}{\lambda_{q+1}} \\
& \left.\stackrel{(84)}{\leq}\left|\int_{\mathbb{T}^{3}}\right| w_{o}\right|^{2} d x-\bar{e}(t) \mid+C \frac{\delta_{q+1}^{1 / 2} \delta_{q}^{1 / 2} \lambda_{q}}{\lambda_{q+1}}+C \frac{\delta_{q+1} \delta_{q}^{1 / 2} \lambda_{q}}{\mu} \\
& \stackrel{(82)}{\leq} C \frac{\delta_{q+1}^{1 / 2} \delta_{q}^{1 / 2} \lambda_{q}}{\lambda_{q+1}}+C \frac{\delta_{q+1} \delta_{q}^{1 / 2} \lambda_{q}}{\mu} .
\end{aligned}
$$

Next, recall that

$$
\begin{aligned}
\bar{e}(t) & =3(2 \pi)^{3} \sum_{l} \chi_{l}^{2} \rho_{l} \\
& =\left(1-\delta_{q+2}\right) \sum_{l} \chi_{l}^{2} e\left(\frac{\mu}{l}\right)-\sum_{l} \chi_{l}^{2} \int_{\mathbb{T}^{3}}\left|v\left(x, l \mu^{-1}\right)\right|^{2} d x .
\end{aligned}
$$

Since $\left|t-\frac{l}{\mu}\right|<\mu^{-1}$ on the support of $\chi_{l}$ and since $\sum_{l} \chi_{l}^{2}=1$, we have

$$
\left|e(t)-\sum_{l} \chi_{l}^{2} e\left(\frac{l}{\mu}\right)\right| \leq \mu^{-1} .
$$

Moreover, using the Euler-Reynolds equation, we can compute

$$
\begin{aligned}
& \int_{\mathbb{T}^{3}}\left(|v(x, t)|^{2}-\left|v\left(x, l \mu^{-1}\right)\right|^{2}\right) d x=\int_{\frac{l}{\mu}}^{t} \int_{\mathbb{T}^{3}} \partial_{t}|v|^{2} \\
= & -\int_{\frac{l}{\mu}}^{t} \int_{\mathbb{T}^{3}} \operatorname{div}\left(v\left(|v|^{2}+2 p\right)\right)+2 \int_{\frac{l}{\mu}}^{t} \int_{\mathbb{T}^{3}} v \cdot \operatorname{div} \stackrel{\circ}{R}=-2 \int_{\frac{l}{\mu}}^{t} \int_{\mathbb{T}^{3}} D v: \stackrel{\circ}{R} .
\end{aligned}
$$


Thus, for $\left|t-\frac{l}{\mu}\right| \leq \mu^{-1}$, we conclude

$$
\left.\left|\int_{\mathbb{T}^{3}}\right| v(x, t)\right|^{2}-\left|v\left(x, l \mu^{-1}\right)\right|^{2} d x \mid \leq C \frac{\delta_{q+1} \delta_{q}^{1 / 2} \lambda_{q}}{\mu} .
$$

Again using $\sum \chi_{l}^{2}=1$, we then conclude

$$
\left|e(t)\left(1-\delta_{q+2}\right)-\left(\bar{e}(t)+\int_{\mathbb{T}^{3}}|v(x, t)|^{2} d x\right)\right| \leq \frac{1}{\mu}+C \frac{\delta_{q+1} \delta_{q}^{1 / 2} \lambda_{q}}{\mu} .
$$

The desired conclusion (80) follows from (85) and (86).

\section{Estimates on the Reynolds stress}

In this section we bound the new Reynolds Stress $\stackrel{\circ}{R}_{1}$. The general pattern in estimating derivatives of the Reynolds stress is that

- the space derivative gets an extra factor of $\lambda_{q+1}$ (when the derivative falls on the exponential factor),

- the transport derivative gets an extra factor $\mu$ (when the derivative falls on the time cutoff).

In fact the transport derivative is slightly more subtle, because in $R^{0}$ a second transport derivative of the perturbation $w$ appears, which leads to an additional term (see (97)). Nevertheless, we organize the estimates in the following proposition according to the above pattern.

Proposition 5.1. For any choice of small positive numbers $\varepsilon$ and $\beta$, there is a constant $C$ (depending only upon these parameters and on e and $M$ ) such that, if $\mu, \lambda_{q+1}$ and $\ell$ satisfy the conditions (59), then we have

$$
\begin{aligned}
& \left\|R^{0}\right\|_{0}+\frac{1}{\lambda_{q+1}}\left\|R^{0}\right\|_{1}+\frac{1}{\mu}\left\|D_{t} R^{0}\right\|_{0} \leq C \frac{\delta_{q+1}^{1 / 2} \mu}{\lambda_{q+1}^{1-\varepsilon}}+\frac{\delta_{q+1}^{1 / 2} \delta_{q} \lambda_{q}}{\lambda_{q+1}^{1-\varepsilon} \mu \ell}, \\
& \left\|R^{1}\right\|_{0}+\frac{1}{\lambda_{q+1}}\left\|R^{1}\right\|_{1}+\frac{1}{\mu}\left\|D_{t} R^{1}\right\|_{0} \leq C \frac{\delta_{q+1} \delta_{q}^{1 / 2} \lambda_{q} \lambda_{q+1}^{\varepsilon}}{\mu}, \\
& \left\|R^{2}\right\|_{0}+\frac{1}{\lambda_{q+1}}\left\|R^{2}\right\|_{1}+\frac{1}{\mu}\left\|D_{t} R^{2}\right\|_{0} \leq C \frac{\delta_{q+1} \delta_{q}^{1 / 2} \lambda_{q}}{\mu}, \\
& \left\|R^{3}\right\|_{0}+\frac{1}{\lambda_{q+1}}\left\|R^{3}\right\|_{1}+\frac{1}{\mu}\left\|D_{t} R^{3}\right\|_{0} \leq C \delta_{q+1}^{1 / 2} \delta_{q}^{1 / 2} \lambda_{q} \ell \\
& \left\|R^{4}\right\|_{0}+\frac{1}{\lambda_{q+1}}\left\|R^{4}\right\|_{1}+\frac{1}{\mu}\left\|D_{t} R^{4}\right\|_{0} \leq C \frac{\delta_{q+1} \delta_{q}^{1 / 2} \lambda_{q}}{\mu}+C \delta_{q+1} \lambda_{q} \ell \\
& \left\|R^{5}\right\|_{0}+\frac{1}{\lambda_{q+1}}\left\|R^{5}\right\|_{1}+\frac{1}{\mu}\left\|D_{t} R^{5}\right\|_{0} \leq C \frac{\delta_{q+1} \delta_{q}^{1 / 2} \lambda_{q}}{\mu} .
\end{aligned}
$$


Thus

$$
\begin{aligned}
\left\|\stackrel{\circ}{R}_{1}\right\|_{0} & +\frac{1}{\lambda_{q+1}}\|\stackrel{\circ}{R}\|_{1}+\frac{1}{\mu}\left\|D_{t} \stackrel{\circ}{R}_{1}\right\|_{0} \\
\leq & C\left(\frac{\delta_{q+1}^{1 / 2} \mu}{\lambda_{q+1}^{1-\varepsilon}}+\frac{\delta_{q+1} \delta_{q}^{1 / 2} \lambda_{q} \lambda_{q+1}^{\varepsilon}}{\mu}+\delta_{q+1}^{1 / 2} \delta_{q}^{1 / 2} \lambda_{q} \ell+\frac{\delta_{q+1}^{1 / 2} \delta_{q} \lambda_{q}}{\lambda_{q+1}^{1-\varepsilon} \mu \ell}\right)
\end{aligned}
$$

and, moreover,

$$
\begin{aligned}
& \left\|\partial_{t} \stackrel{\circ}{R}_{1}+v_{1} \cdot \nabla \stackrel{\circ}{R}_{1}\right\|_{0} \\
\leq & C \delta_{q+1}^{1 / 2} \lambda_{q+1}\left(\frac{\delta_{q+1}^{1 / 2} \mu}{\lambda_{q+1}^{1-\varepsilon}}+\frac{\delta_{q+1} \delta_{q}^{1 / 2} \lambda_{q} \lambda_{q+1}^{\varepsilon}}{\mu}+\delta_{q+1}^{1 / 2} \delta_{q}^{1 / 2} \lambda_{q} \ell+\frac{\delta_{q+1}^{1 / 2} \delta_{q} \lambda_{q}}{\lambda_{q+1}^{1-\varepsilon} \mu \ell}\right) .
\end{aligned}
$$

Proof. Estimates on $R^{0}$. We start by calculating

$$
\partial_{t} w+v_{\ell} \cdot \nabla w+w \cdot \nabla v_{\ell}=\sum_{k l}\left(\chi_{l}^{\prime} L_{k l}+\chi_{l} D_{t} L_{k l}+\chi_{l} L_{k l} \cdot \nabla v_{\ell}\right) e^{i k \cdot \Phi_{l}} .
$$

Define $\Omega_{k l}:=\left(\chi_{l}^{\prime} L_{k l}+\chi_{l} D_{t} L_{k l}+\chi_{l} L_{k l} \cdot \nabla v_{\ell}\right) \phi_{k l}$, and write (recalling the identity $\left.\phi_{k l} e^{i \lambda_{q+1} k \cdot x}=e^{i \lambda_{q+1} k \cdot \Phi_{l}}\right)$

$$
\partial_{t} w+v_{\ell} \cdot \nabla w+w \cdot \nabla v_{\ell}=\sum_{k l} \Omega_{k l} e^{i \lambda_{q+1} k \cdot x} .
$$

Using Lemmas 3.1 and 3.2 and (60),

$$
\left\|\Omega_{k l}\right\|_{0} \leq C \delta_{q+1}^{1 / 2} \mu\left(1+\frac{\delta_{q}^{1 / 2} \lambda_{q}}{\mu}\right) \leq C \delta_{q+1}^{1 / 2} \mu
$$

and similarly, for $N \geq 1$,

$$
\left\|\Omega_{k l}\right\|_{N} \leq C \delta_{q+1}^{1 / 2} \mu\left(\ell^{-N}+\left\|\phi_{k l}\right\|_{N}\right) \leq C \delta_{q+1}^{1 / 2} \mu \lambda_{q+1}^{N(1-\beta)} .
$$

Moreover, observe that although this estimate has been derived for $N$ integer, by the interpolation inequality (128) it can be easily extended to any real $N \geq 1$. (Besides, this fact will be used frequently in the rest of the proof.) Applying Proposition G.1(ii) we obtain

$$
\begin{aligned}
\left\|R_{0}\right\|_{0} & \leq \sum_{k l}\left(\lambda_{q+1}^{\varepsilon-1}\left\|\Omega_{k l}\right\|_{0}+\lambda_{q+1}^{-N+\varepsilon}\left[\Omega_{k l}\right]_{N}+\lambda_{q+1}^{-N}\left[\Omega_{k l}\right]_{N+\varepsilon}\right) \\
& \leq C \delta_{q+1}^{1 / 2} \mu\left(\lambda_{q+1}^{-1+\varepsilon}+\lambda_{q+1}^{-N \beta+\varepsilon}\right) .
\end{aligned}
$$

It suffices to choose $N$ so that $N \beta \geq 1$ in order to achieve

$$
\left\|R^{0}\right\|_{0} \leq C \delta_{q+1}^{1 / 2} \mu \lambda_{q+1}^{\varepsilon-1} .
$$

As for $\left\|R^{0}\right\|_{1}$, we differentiate (95). We therefore conclude

$$
\partial_{j} R^{0}=\mathcal{R}\left(\sum_{k l}\left(i \lambda_{q+1} k_{j} \Omega_{k l}+\partial_{j} \Omega_{k l}\right) e^{i \lambda_{q+1} k \cdot x}\right) .
$$


Applying Proposition G.1(ii) as before, we conclude $\left\|R^{0}\right\|_{1} \leq C \delta_{q+1}^{1 / 2} \mu \lambda_{q+1}^{\varepsilon}$.

Estimates on $D_{t} R^{0}$. We start by calculating

$$
\begin{aligned}
& D_{t}\left(\partial_{t} w+v_{\ell} \cdot \nabla w+w \cdot \nabla v_{\ell}\right)=\sum_{k l}\left(\partial_{t}^{2} \chi_{l} L_{k l}+2 \partial_{t} \chi_{l} D_{t} L_{k l}+\chi_{l} D_{t}^{2} L_{k l}\right. \\
& \left.+\partial_{t} \chi_{l} L_{k l} \cdot D v_{\ell}+\chi_{l} D_{t} L_{k l} \cdot D v_{\ell}+\chi_{l} L_{k l} \cdot D D_{t} v_{\ell}-\chi_{l} L_{k l} \cdot D v_{\ell} \cdot D v_{\ell}\right) e^{i k \cdot \Phi_{l}} \\
= & \sum_{k l} \Omega_{k l}^{\prime} e^{i \lambda_{q+1} k \cdot x} .
\end{aligned}
$$

As before, we have

$$
\left\|\Omega_{k l}^{\prime}\right\|_{0} \leq C \delta_{q+1}^{1 / 2} \mu\left(\mu+\frac{\delta_{q} \lambda_{q}}{\mu \ell}+\delta_{q}^{1 / 2} \lambda_{q}+\frac{\delta_{q} \lambda_{q}^{2}}{\mu}\right) \leq C \delta_{q+1}^{1 / 2} \mu\left(\mu+\frac{\delta_{q} \lambda_{q}}{\mu \ell}\right)
$$

and, for any $N \geq 1$,

$$
\begin{aligned}
\left\|\Omega_{k l}^{\prime}\right\|_{N} & \leq C \delta_{q+1}^{1 / 2} \mu \ell^{-N}\left(\mu+\frac{\delta_{q} \lambda_{q}}{\mu \ell}+\delta_{q}^{1 / 2} \lambda_{q}+\frac{\delta_{q} \lambda_{q}^{2}}{\mu}\right)+\left\|\Omega_{k l}^{\prime}\right\|_{0}\left\|\phi_{k l}\right\|_{N} \\
& \leq C \delta_{q+1}^{1 / 2} \mu\left(\mu+\frac{\delta_{q} \lambda_{q}}{\mu \ell}\right)\left(\ell^{-N}+\left\|\phi_{k l}\right\|_{N}\right) \\
& \leq C \delta_{q+1}^{1 / 2} \mu\left(\mu+\frac{\delta_{q} \lambda_{q}}{\mu \ell}\right) \lambda_{q+1}^{N(1-\beta)} .
\end{aligned}
$$

Next, observe that we can write

$$
\begin{aligned}
D_{t} R^{0} & =\left(\left[D_{t}, \mathcal{R}\right]+\mathcal{R} D_{t}\right)\left(\partial_{t} w+v_{\ell} \cdot \nabla w+w \cdot \nabla v_{\ell}\right) \\
& =\left(\left[v_{\ell}, \mathcal{R}\right] D+\mathcal{R} D_{t}\right)\left(\partial_{t} w+v_{\ell} \cdot \nabla w+w \cdot \nabla v_{\ell}\right)
\end{aligned}
$$

as is customary, $[A, B]$ denotes the commutator $A B-B A$ of two operators $A$ and $B$; the operator $\left[v_{\ell}, \mathcal{R}\right]$ is applied to derivatives $D \Lambda$ of tensors $\Lambda$, and it gives

$$
\left[v_{\ell}, \mathcal{R}\right] D \Lambda=\sum_{j=1}^{3}\left[\left(v_{\ell}\right)_{j} \mathcal{R}\left(\frac{\partial \Lambda}{\partial x_{j}}\right)-\mathcal{R}\left(\left(v_{\ell}\right)_{j} \frac{\partial \Lambda}{\partial x_{j}}\right)\right]
$$

Using this convention we then compute

$$
\begin{aligned}
D_{t} R^{0}=\sum_{k l}( & {\left[v_{\ell} \cdot \mathcal{R}\right]\left(D \Omega_{k l} e^{i \lambda_{q+1} k \cdot x}\right) } \\
& \left.+i \lambda_{q+1}\left[v_{\ell} \cdot k, \mathcal{R}\right]\left(\Omega_{k l} e^{i \lambda_{q+1} k \cdot x}\right)+\mathcal{R}\left(\Omega_{k l}^{\prime} e^{i \lambda_{q+1} k \cdot x}\right)\right) .
\end{aligned}
$$


Using the estimates for $\Omega_{k l}^{\prime}$ derived above, and applying Proposition G.1(ii), we obtain

$$
\begin{aligned}
\left\|\mathcal{R}\left(\Omega_{k l}^{\prime} e^{i \lambda_{q+1} k \cdot x}\right)\right\|_{0} & \leq \frac{\left\|\Omega_{k l}^{\prime}\right\|_{0}}{\lambda_{q+1}^{1-\varepsilon}}+\frac{\left[\Omega_{k l}^{\prime}\right]_{N}}{\lambda_{q+1}^{N-\varepsilon}}+\frac{\left[\Omega_{k l}^{\prime}\right]_{N+\varepsilon}}{\lambda_{q+1}^{N}} \\
& \leq C \frac{\delta_{q+1}^{1 / 2} \mu}{\lambda_{q+1}^{1-\varepsilon}}\left(\mu+\frac{\delta_{q} \lambda_{q}}{\mu \ell}\right)\left(1+\lambda_{q+1}^{1-N \beta}\right) .
\end{aligned}
$$

Furthermore, applying Proposition H.1 we obtain

$$
\begin{aligned}
& \left\|\left[v_{\ell}, \mathcal{R}\right]\left(D \Omega_{k l} e^{i \lambda_{q+1} k \cdot x}\right)\right\|_{0} \leq \frac{C}{\lambda_{q+1}^{2-\varepsilon}}\left\|v_{\ell}\right\|_{1}\left\|\Omega_{k l}\right\|_{1} \\
& +\frac{C}{\lambda_{q+1}^{N-\varepsilon}} \sum_{i=0}^{N-1}\left\|\Omega_{k l}\right\|_{1+i+\varepsilon}\left\|v_{\ell}\right\|_{N-i+\varepsilon} \leq C \frac{\delta_{q}^{1 / 2} \lambda_{q} \delta_{q+1}^{1 / 2} \mu}{\lambda_{q+1}^{1-\varepsilon}}\left(\lambda_{q+1}^{-\beta}+\lambda_{q+1}^{1+2 \varepsilon-N \beta}\right)
\end{aligned}
$$

and, similarly,

$$
\lambda_{q+1}\left\|\left[v_{\ell} \cdot k, \mathcal{R}\right]\left(\Omega_{k l} e^{i \lambda_{q+1} k \cdot x}\right)\right\|_{0} \leq C \frac{\delta_{q}^{1 / 2} \lambda_{q} \delta_{q+1}^{1 / 2} \mu}{\lambda_{q+1}^{1-\varepsilon}}\left(1+\lambda_{q+1}^{1+2 \varepsilon-N \beta}\right) .
$$

By choosing $N \in \mathbb{N}$ sufficiently large so that $N \beta \geq 1+2 \varepsilon$, we deduce

$$
\left\|D_{t} R^{0}\right\|_{0} \leq C \frac{\delta_{q+1}^{1 / 2} \mu}{\lambda_{q+1}^{1-\varepsilon}}\left(\mu+\delta_{q}^{1 / 2} \lambda_{q}+\frac{\delta_{q} \lambda_{q}}{\mu \ell}\right)
$$

Taking into account that $\delta_{q}^{1 / 2} \lambda_{q} \leq \mu$, this concludes the proof of (87).

Remark 3. The estimate on $D_{t} R_{t}^{0}$ could be improved by keeping track, along the iteration, of $\left\|p_{q}\right\|_{2}$ and $\left\|\stackrel{\circ}{R}_{q}\right\|_{2}$ : such quantities can indeed be used to get a better control of $\left\|D_{t} v\right\|_{1}$. This observation, which is used already in [18], plays an important role in [1] (cf. Section 7 below).

Estimates on $R^{1}$. Using Lemma 2.1, we have

$$
\begin{aligned}
\operatorname{div}\left(w_{o} \otimes w_{o}\right. & \left.-\sum_{l} \chi_{l}^{2} \stackrel{\circ}{R}_{\ell, l}-\frac{\left|w_{o}\right|^{2}}{2} \mathrm{Id}\right) \\
& =\sum_{\substack{(k, l),\left(k^{\prime}, l^{\prime}\right) \\
k+k^{\prime} \neq 0}} \chi_{l} \chi_{l^{\prime}} \operatorname{div}\left(w_{k l} \otimes w_{k^{\prime} l^{\prime}}-\frac{w_{k l} \cdot w_{k^{\prime} l^{\prime}}}{2} \mathrm{Id}\right)=I+I I
\end{aligned}
$$


where, setting $f_{k l k^{\prime} l^{\prime}}:=\chi_{l} \chi_{l^{\prime}} a_{k l} a_{k^{\prime} l^{\prime}} \phi_{k l} \phi_{k^{\prime} l^{\prime}}$,

$$
\begin{gathered}
I=\sum_{\substack{(k, l),\left(k^{\prime}, l^{\prime}\right) \\
k+k^{\prime} \neq 0}}\left(B_{k} \otimes B_{k^{\prime}}-\frac{1}{2}\left(B_{k} \cdot B_{k^{\prime}}\right) \mathrm{Id}\right) \nabla f_{k l k^{\prime} l^{\prime}} e^{i \lambda_{q+1}\left(k+k^{\prime}\right) \cdot x}, \\
I I=i \lambda_{q+1} \sum_{\substack{(k, l),\left(k^{\prime}, l^{\prime}\right) \\
k+k^{\prime} \neq 0}} f_{k l k^{\prime} l^{\prime}}\left(B_{k} \otimes B_{k^{\prime}}-\frac{1}{2}\left(B_{k} \cdot B_{k^{\prime}}\right) \mathrm{Id}\right)\left(k+k^{\prime}\right) e^{i \lambda_{q+1}\left(k+k^{\prime}\right) \cdot x} .
\end{gathered}
$$

Concerning $I I$, recall that the summation is over all $l \in \mathbb{Z} \cap[0, \mu]$ and all $k \in \Lambda^{e}$ if $l$ is even and all $k \in \Lambda^{o}$ if $l$ is odd. Furthermore, both $\Lambda^{e}, \Lambda^{o} \subset \bar{\lambda} S^{2} \cap \mathbb{Z}^{3}$ satisfy the conditions of Lemma 1.3. Therefore we may symmetrize the summand in $I I$ in $k$ and $k^{\prime}$. On the other hand, recall from Lemma 1.2 that

$$
\left(B_{k} \otimes B_{k^{\prime}}+B_{k^{\prime}} \otimes B_{k}\right)\left(k+k^{\prime}\right)=\left(B_{k} \cdot B_{k^{\prime}}\right)\left(k+k^{\prime}\right) .
$$

From this we deduce that $I I=0$.

Concerning $I$, we first note, using the product rule, (64) and (65), that

$$
\left[f_{k l k^{\prime} l^{\prime}}\right]_{N} \leq C \delta_{q+1}\left(\lambda_{q} \ell^{1-N}+\left\|\phi_{k l} \phi_{k^{\prime} l^{\prime}}\right\|_{N}\right) \quad \text { for } N \geq 1 \text {. }
$$

By Lemma (67) and (59) (cf. (60)) we then conclude

$$
\begin{aligned}
& {\left[f_{k l k^{\prime} l^{\prime}}\right]_{1} \leq C \delta_{q+1}\left(\lambda_{q}+\lambda_{q+1} \frac{\delta_{q}^{1 / 2} \lambda_{q}}{\mu}\right) \leq C \delta_{q+1} \lambda_{q+1} \frac{\delta_{q}^{1 / 2} \lambda_{q}}{\mu},} \\
& {\left[f_{k l k^{\prime} l^{\prime}}\right]_{N} \leq C \delta_{q+1} \lambda_{q+1}^{N(1-\beta)}, \quad N \geq 2 .}
\end{aligned}
$$

Applying Proposition G.1(ii) to $I$, we obtain

$$
\begin{aligned}
\left\|R^{1}\right\|_{0} \leq & \sum_{\substack{(k, l),\left(k^{\prime}, l^{\prime}\right) \\
k+k^{\prime} \neq 0}}\left(\lambda_{q+1}^{\varepsilon-1}\left[f_{k l k^{\prime} l^{\prime}}\right]_{1}+\lambda_{q+1}^{-N+\varepsilon}\left[f_{k l k^{\prime} l^{\prime}}\right]_{N+1}+\lambda_{q+1}^{-N}\left[f_{k l k^{\prime} l^{\prime}}\right]_{N+1+\varepsilon}\right) \\
& \leq C \delta_{q+1}\left(\lambda_{q+1}^{\varepsilon} \frac{\delta_{q}^{1 / 2} \lambda_{q}}{\mu}+\lambda_{q+1}^{1-N \beta+\varepsilon}\right) .
\end{aligned}
$$

By choosing $N$ sufficiently large, we deduce

$$
\left\|R^{1}\right\|_{0} \leq C \frac{\delta_{q+1} \delta_{q}^{1 / 2} \lambda_{q} \lambda_{q+1}^{\varepsilon}}{\mu}
$$

as required. Moreover, differentiating we conclude $\partial_{j} R^{1}=\mathcal{R}\left(\partial_{j} I\right)$ where

$$
\begin{aligned}
\partial_{j} I= & \sum_{\substack{k, l),\left(k^{\prime}, l^{\prime}\right) \\
k+k^{\prime} \neq 0}}\left(B_{k} \otimes B_{k^{\prime}}-\frac{1}{2}\left(B_{k} \cdot B_{k^{\prime}}\right) \mathrm{Id}\right) \\
& \cdot\left(i \lambda_{q+1}\left(k+k^{\prime}\right)_{j} \nabla f_{k l k^{\prime} l^{\prime}}+\partial_{j} \nabla f_{k l k^{\prime} l^{\prime}}\right) e^{i \lambda_{q+1}\left(k+k^{\prime}\right) \cdot x} .
\end{aligned}
$$

Therefore we again apply Proposition G.1(ii) to conclude the desired estimate for $\left\|R^{1}\right\|_{1}$. 
Estimates on $D_{t} R^{1}$. As in the estimate for $D_{t} R^{0}$, we again make use of the identity $D_{t} \mathcal{R}=\left[v_{\ell}, \mathcal{R}\right] D+\mathcal{R} D_{t}$ in order to write

$$
\begin{aligned}
& D_{t} R^{1}=\sum_{\substack{(k, l),\left(k^{\prime}, l^{\prime}\right) \\
k+k^{\prime} \neq 0}}\left(\left[v_{\ell}, \mathcal{R}\right]\left(D U_{k l k^{\prime} l^{\prime}} e^{i \lambda_{q+1}\left(k+k^{\prime}\right) \cdot x}\right)\right. \\
& \left.+i \lambda_{q+1}\left[v_{\ell} \cdot\left(k+k^{\prime}\right), \mathcal{R}\right]\left(U_{k l k^{\prime} l^{\prime}} e^{i \lambda_{q+1}\left(k+k^{\prime}\right) \cdot x}\right)+\mathcal{R}\left(U_{k l k^{\prime} l^{\prime}}^{\prime} e^{i \lambda_{q+1}\left(k+k^{\prime}\right) \cdot x}\right)\right),
\end{aligned}
$$

where we have set $U_{k l k^{\prime} l^{\prime}}=\left(B_{k} \otimes B_{k^{\prime}}-\frac{1}{2}\left(B_{k} \cdot B_{k^{\prime}}\right) \mathrm{Id}\right) \nabla f_{k l k^{\prime} l^{\prime}}$ and

$$
D_{t} \operatorname{div}\left(w_{o} \otimes w_{o}-\sum_{l} \chi_{l}^{2} \stackrel{\circ}{R}_{\ell, l}-\frac{\left|w_{o}\right|^{2}}{2} \mathrm{Id}\right)=\sum_{\substack{(k, l),\left(k^{\prime}, l^{\prime}\right) \\ k+k^{\prime} \neq 0}} U_{k l k^{\prime} l^{\prime}}^{\prime} e^{i \lambda_{q+1}\left(k+k^{\prime}\right) \cdot x} .
$$

In order to further compute $U_{k l k^{\prime} l^{\prime}}^{\prime}$, we write

$$
\begin{aligned}
& \nabla f_{k l k^{\prime} l^{\prime}} e^{i \lambda_{q+1}\left(k+k^{\prime}\right) \cdot x}=\chi_{l} \chi_{l^{\prime}}\left(a_{k l} \nabla a_{k^{\prime} l^{\prime}}+a_{k^{\prime} l^{\prime}} \nabla a_{k l}\right) e^{i \lambda_{q+1}\left(k \cdot \Phi_{l}+k^{\prime} \cdot \Phi_{l^{\prime}}\right)} \\
& \quad+i \lambda_{q+1} \chi_{l} \chi_{l^{\prime}} a_{k l} a_{k^{\prime} l^{\prime}}\left(\left(D \Phi_{l}-\mathrm{Id}\right) k+\left(D \Phi_{l^{\prime}}-\mathrm{Id}\right) k^{\prime}\right) e^{i \lambda_{q+1}\left(k \cdot \Phi_{l}+k^{\prime} \cdot \Phi_{l^{\prime}}\right)}
\end{aligned}
$$

and hence, using (79),

$$
\begin{aligned}
D_{t} & \left(\nabla f_{k l k^{\prime} l^{\prime}} e^{i \lambda_{q+1}\left(k+k^{\prime}\right) \cdot x}\right)=\left(\chi_{l} \chi_{l^{\prime}}\right)^{\prime}\left(a_{k l} \nabla a_{k^{\prime} l^{\prime}}+a_{k^{\prime} l^{\prime}} \nabla a_{k l}\right) e^{i \lambda_{q+1}\left(k \cdot \Phi_{l}+k^{\prime} \cdot \Phi_{l^{\prime}}\right)} \\
& +i \lambda_{q+1}\left(\chi_{l} \chi_{l^{\prime}}\right)^{\prime} a_{k l} a_{k^{\prime} l^{\prime}}\left(\left(D \Phi_{l}-\mathrm{Id}\right) k+\left(D \Phi_{l^{\prime}}-\mathrm{Id}\right) k^{\prime}\right) e^{i \lambda_{q+1}\left(k \cdot \Phi_{l}+k^{\prime} \cdot \Phi_{l^{\prime}}\right)} \\
& -\chi_{l} \chi_{l^{\prime}}\left(a_{k l} D v_{\ell}^{T} \nabla a_{k^{\prime} l^{\prime}}+a_{k^{\prime} l^{\prime}} D v_{\ell}^{T} \nabla a_{k l}\right) e^{i \lambda_{q+1}\left(k \cdot \Phi_{l}+k^{\prime} \cdot \Phi_{l^{\prime}}\right)} \\
& -i \lambda_{q+1} \chi_{l} \chi_{l^{\prime}} a_{k l} a_{k^{\prime} l^{\prime}}\left(D \Phi_{l} D v_{\ell}^{T} k+D \Phi_{l^{\prime}} D v_{\ell}^{T} k^{\prime}\right) e^{i \lambda_{q+1}\left(k \cdot \Phi_{l}+k^{\prime} \cdot \Phi_{l^{\prime}}\right)} \\
=: & \left(\Sigma_{k l k^{\prime} l^{\prime}}^{1}+\Sigma_{k l k^{\prime} l^{\prime}}^{2}+\Sigma_{k l k^{\prime} l^{\prime}}^{3}+\Sigma_{k l k^{\prime} l^{\prime}}^{4}\right) e^{i \lambda_{q+1}\left(k \cdot \Phi_{l}+k^{\prime} \cdot \Phi_{l^{\prime}}\right)} \\
=: & \Sigma_{k l k^{\prime} l^{\prime}} e^{i \lambda_{q+1}\left(k \cdot \Phi_{l}+k^{\prime} \cdot \Phi_{l^{\prime}}\right)} .
\end{aligned}
$$

Ignoring the subscripts we can use (127), Lemma 3.1 and Lemma 3.2 to estimate

$$
\begin{aligned}
\|\Sigma\|_{N} & \leq C \delta_{q+1} \lambda_{q} \ell^{-N}\left(\mu+\lambda_{q+1} \delta_{q}^{1 / 2}+\delta_{q}^{1 / 2} \lambda_{q}+\lambda_{q+1} \delta_{q}^{1 / 2}\right) \\
& \stackrel{(60)}{\leq} C \delta_{q+1} \lambda_{q+1} \delta_{q}^{1 / 2} \lambda_{q} \ell^{-N} .
\end{aligned}
$$

We thus conclude

$$
\begin{aligned}
\left\|U_{k l k^{\prime} l^{\prime}}^{\prime}\right\|_{N} & \leq C\left\|\Sigma_{k l k^{\prime} l^{\prime}}\right\|_{N}+C\left\|\Sigma_{k l k^{\prime} l^{\prime}}\right\|_{0}\left\|\phi_{k l} \phi_{k^{\prime} l^{\prime}}\right\|_{N} \\
& \leq C \delta_{q+1} \lambda_{q+1} \delta_{q}^{1 / 2} \lambda_{q}\left(\ell^{-N}+\lambda_{q+1}^{N(1-\beta)}\right) \leq C \delta_{q+1} \delta_{q}^{1 / 2} \lambda_{q} \lambda_{q+1}^{1+N(1-\beta)} .
\end{aligned}
$$

The estimate on $\left\|D_{t} R^{1}\right\|_{0}$ now follows exactly as above for $D_{t} R^{0}$ applying Proposition H.1 to the commutator terms. This concludes the verification of $(88)$. 
Estimates on $R^{2}$ and $D_{t} R^{2}$. Using Lemma 3.1, we have

$$
\begin{aligned}
& \left\|R^{2}\right\|_{0} \leq C\left(\left\|w_{c}\right\|_{0}^{2}+\left\|w_{o}\right\|_{0}\left\|w_{c}\right\|_{0}\right) \leq C \delta_{q+1} \frac{\delta_{q}^{1 / 2} \lambda_{q}}{\mu} \\
& \left\|R^{2}\right\|_{1} \leq C\left(\left\|w_{c}\right\|_{1}\left\|w_{c}\right\|_{0}+\left\|w_{o}\right\|_{1}\left\|w_{c}\right\|_{0}+\left\|w_{o}\right\|_{0}\left\|w_{c}\right\|_{1}\right) \leq C \delta_{q+1} \frac{\delta_{q}^{1 / 2} \lambda_{q}}{\mu} \lambda_{q+1} .
\end{aligned}
$$

Similarly, with the Lemmas 3.1 and 3.2 we achieve

$$
\left\|D_{t} R^{2}\right\|_{0} \leq C\left\|D_{t} w_{c}\right\|_{0}\left(\left\|w_{o}\right\|_{0}+\left\|w_{c}\right\|_{0}\right)+C\left\|D_{t} w_{o}\right\|_{0}\left\|w_{c}\right\|_{0} \leq C \delta_{q+1} \delta_{q}^{1 / 2} \lambda_{q} .
$$

Estimates on $R^{3}$ and $D_{t} R^{3}$. The estimates on $\left\|R^{3}\right\|_{0}$ and $\left\|R^{3}\right\|_{1}$ are a direct consequence of the mollification estimates (30) and (32) as well as Lemma 3.1. Moreover,

$$
\begin{aligned}
\left\|D_{t} R^{3}\right\|_{0} & \leq\left\|v-v_{\ell}\right\|_{0}\left\|D_{t} w\right\|_{0}+\left(\left\|D_{t} v\right\|_{0}+\left\|D_{t} v_{\ell}\right\|\right)\|w\|_{0} \\
& =\left\|v-v_{\ell}\right\|_{0}\left(\left\|D_{t} w_{c}\right\|_{0}+\left\|D_{t} w_{o}\right\|\right)+\left(\left\|D_{t} v\right\|_{0}+\left\|D_{t} v_{\ell}\right\|\right)\|w\|_{0} .
\end{aligned}
$$

Concerning $D_{t} v$, note that, by our inductive hypothesis

$$
\begin{aligned}
\left\|D_{t} v\right\|_{0} & \leq\left\|\partial_{t} v+v \cdot \nabla v\right\|_{0}+\left\|v-v_{\ell}\right\|_{0}\|v\|_{1} \\
& \leq\left\|p_{q}\right\|_{1}+\left\|\stackrel{\circ}{R}_{q}\right\|_{1}+C \delta_{q} \lambda_{q}^{2} \ell \leq C \delta_{q} \lambda_{q} .
\end{aligned}
$$

Thus the required estimate on $D_{t} R^{3}$ follows from Lemma 3.2.

Estimates on $R^{4}$ and $D_{t} R^{4}$. From the mollification estimates (31) and (33), we deduce

$$
\begin{aligned}
& \left\|R^{4}\right\|_{0} \leq C\|\stackrel{\circ}{R}\|_{1} \ell \leq C \delta_{q+1} \lambda_{q} \ell, \\
& \left\|R^{4}\right\|_{1} \leq 2\|\stackrel{\circ}{R}\|_{1} \leq C \delta_{q+1} \lambda_{q} .
\end{aligned}
$$

As for $D_{t} R^{4}$, observe first that, using our inductive hypothesis,

$$
\left\|D_{t} \stackrel{\circ}{R}\right\|_{0} \leq\left\|\partial_{t} \stackrel{\circ}{R}+v \cdot \nabla \stackrel{\circ}{R}\right\|_{0}+\left\|v_{\ell}-v\right\|_{0}\|\stackrel{\circ}{R}\|_{1} \leq C \delta_{q+1} \delta_{q}^{1 / 2} \lambda_{q}+C \delta_{q+1} \delta_{q}^{1 / 2} \lambda_{q}^{2} \ell .
$$

Moreover,

$$
\begin{aligned}
D_{t} \stackrel{\circ}{R}_{\ell} & =\left(D_{t} \stackrel{\circ}{R}\right) * \psi_{\ell}+v_{\ell} \cdot \nabla \stackrel{\circ}{R}_{\ell}-\left(v_{\ell} \cdot \nabla \stackrel{\circ}{R}\right) * \psi_{\ell} \\
& =\left(D_{t} \stackrel{\circ}{R}\right) * \psi_{\ell}+\operatorname{div}\left(v_{\ell} \otimes \stackrel{\circ}{R}_{\ell}-(v \otimes \stackrel{\circ}{R}) * \psi_{\ell}\right)+\left[\left(v-v_{\ell}\right) \cdot \nabla \stackrel{\circ}{R}\right] * \psi_{\ell},
\end{aligned}
$$

where we have used that $\operatorname{div} v=0$. Using Proposition E.1, we deduce

$$
\left\|v_{\ell} \otimes \stackrel{\circ}{R}_{\ell}-(v \otimes \stackrel{\circ}{R}) * \psi_{\ell}\right\|_{1} \leq C \delta_{q+1} \delta_{q}^{1 / 2} \lambda_{q} \lambda_{q} \ell .
$$

Gathering all the estimates we then achieve

$$
\left\|D_{t} R^{4}\right\|_{0} \leq\left\|D_{t} \stackrel{\circ}{R}\right\|_{0}+\left\|D_{t} \stackrel{\circ}{R}_{\ell}\right\|_{0} \leq C \delta_{q+1} \lambda_{q} \delta_{q}^{1 / 2} .
$$

The estimate (91) follows now using (60). 
Estimates on $R^{5}$. Recall that $D_{t} \stackrel{\circ}{R}_{\ell, l}=0$. Therefore, using the arguments from (102),

$$
\left\|D_{t}\left(\stackrel{\circ}{R}_{\ell}-\stackrel{\circ}{R}_{\ell, l}\right)\right\|_{0}=\left\|D_{t} \stackrel{\circ}{R}_{\ell}\right\|_{0} \leq C \delta_{q+1} \delta_{q}^{1 / 2} \lambda_{q} .
$$

On the other hand, using again the identity (102) and Proposition E.1,

$$
\begin{aligned}
\left\|D_{t}\left(\stackrel{\circ}{R}_{\ell}-\stackrel{\circ}{R}_{\ell, l}\right)\right\|_{1} & =\left\|D_{t} \stackrel{\circ}{R}_{\ell}\right\|_{1} \leq C \ell^{-1}\left\|D_{t} \stackrel{\circ}{R}\right\|_{0}+\left\|v_{\ell} \otimes \stackrel{\circ}{R}_{\ell}-(v \otimes \stackrel{\circ}{R}) * \psi_{\ell}\right\|_{2} \\
& +C \ell^{-1}\left\|v-v_{\ell}\right\|_{0}\|\stackrel{\circ}{R}\|_{1} \leq C \delta_{q+1} \delta_{q}^{1 / 2} \lambda_{q} \ell^{-1} .
\end{aligned}
$$

Since $\stackrel{\circ}{R}_{\ell, l}\left(x, t \mu^{-1}\right)=\stackrel{\circ}{R}_{\ell}\left(x, t \mu^{-1}\right)$, the difference $\stackrel{\circ}{R}_{\ell}-\stackrel{\circ}{R}_{\ell, l}$ vanishes at $t=l \mu^{-1}$. From Proposition D.1 we deduce that, for times $t$ in the support of $\chi_{l}$ (i.e., $\left.\left|t-l \mu^{-1}\right|<\mu^{-1}\right)$,

$$
\begin{aligned}
& \left\|\stackrel{\circ}{R}_{\ell}-\stackrel{\circ}{R}_{\ell, l}\right\|_{0} \leq C \mu^{-1}\left\|D_{t}\left(\stackrel{\circ}{R}_{\ell}-\stackrel{\circ}{R}_{\ell, l}\right)\right\|_{0} \leq C \mu^{-1} \delta_{q+1} \delta_{q}^{1 / 2} \lambda_{q}, \\
& \left\|\stackrel{\circ}{R}_{\ell}-\stackrel{\circ}{R}_{\ell, l}\right\|_{1} \leq C \mu^{-1}\left\|D_{t}\left(\stackrel{\circ}{R}_{\ell}-\stackrel{\circ}{R}_{\ell, l}\right)\right\|_{1} \leq C \mu^{-1} \delta_{q+1} \delta_{q}^{1 / 2} \lambda_{q} \ell^{-1} .
\end{aligned}
$$

The desired estimates on $\left\|R^{5}\right\|_{0}$ and $\left\|R^{5}\right\|_{1}$ then follow easily using (60).

Estimate on $D_{t} R^{5}$. In this case we compute

$$
D_{t} R^{5}=\sum_{l} 2 \chi_{l} \partial_{t} \chi_{l}\left(\stackrel{\circ}{R}_{\ell}-\stackrel{\circ}{R}_{\ell, l}\right)+\sum_{l} \chi_{l}^{2} D_{t} \stackrel{\circ}{R}_{\ell}
$$

The second summand has been estimated above and, since $\left\|\partial_{t} \chi_{l}\right\|_{0} \leq C \mu$, the first summand can be estimated by $C \mu \delta_{q+1} \delta_{q}^{1 / 2} \lambda_{q} \mu^{-1}$ (again appealing to the arguments above).

Proof of (94). To achieve this last inequality, observe that

$$
\left\|\partial_{t} \stackrel{\circ}{R}_{1}+v_{1} \cdot \nabla \stackrel{\circ}{R}_{1}\right\|_{0} \leq\left\|D_{t} \stackrel{\circ}{R}_{1}\right\|_{0}+\left(\left\|v-v_{\ell}\right\|_{0}+\|w\|_{0}\right)\left\|\stackrel{\circ}{R}_{1}\right\|_{1} .
$$

On the other hand, by (30) and (59), $\left\|v-v_{\ell}\right\|_{0} \leq C \delta_{q}^{1 / 2} \lambda_{q} \ell \leq \delta_{q+1}^{1 / 2}$. Moreover, by (50), (68) and (60), $\|w\| \leq\left\|w_{o}\right\|_{0}+\left\|w_{c}\right\|_{0} \leq C \delta_{q+1}^{1 / 2}$. Thus, by (93) we conclude

$$
\begin{aligned}
& \left\|\partial_{t} \stackrel{\circ}{R}_{1}+v_{1} \cdot \nabla \stackrel{\circ}{R}_{1}\right\|_{0} \leq C\left(\mu+\delta_{q+1}^{1 / 2} \lambda_{q+1}\right) \\
& \quad\left(\frac{\delta_{q+1}^{1 / 2} \mu}{\lambda_{q+1}^{1-\varepsilon}}+\frac{\delta_{q+1} \delta_{q}^{1 / 2} \lambda_{q} \lambda_{q+1}^{\varepsilon}}{\mu}+\delta_{q+1}^{1 / 2} \delta_{q}^{1 / 2} \lambda_{q} \ell+\frac{\delta_{q+1}^{1 / 2} \delta_{q} \lambda_{q}}{\lambda_{q+1}^{1-\varepsilon} \mu \ell}\right) .
\end{aligned}
$$

Since by (60) $\mu \leq \delta_{q+1}^{1 / 2} \lambda_{q+1}$, (94) follows easily.

5.1. A remark on the bounds for $R^{5}$ and $D_{t} R^{0}$. By approximating the error with the solution to a transport equation, we have saved a lot of work in obtaining the estimate for $\left\|D_{t} R^{0}\right\|$ in (87) compared to the approach of [18]. The price we have paid here is introducing the additional error term $R^{5}$, which represents a new degree of freedom in the construction that had not previously been exploited. The estimate (92) for $R^{5}$ is among the maximal bounds in the argument and it is thus one of the obstructions to going beyond $\frac{1}{5}$. It should 
be noticed that it is not clear which error terms must be improved in order to reach the conjectural exponent $\frac{1}{3}$. For instance, in the paper [1] (cf. Section 7) the error estimate (92) is not an obstruction to get the Hölder regularity in space on the set of "good times." On the other hand, in the approach of [18] such an error term does not appear, and it is of interest to review it here.

The difficulty in bounding $D_{t} R^{0}$ is that the transport term $R^{0}$ by itself involves the advective derivative of the amplitudes $a_{k}$, which in turn depend on the input stress $\stackrel{\circ}{R}$ by (39). Consequently, $D_{t} R^{0}$ essentially involves the second advective derivative $D_{t}^{2} \stackrel{\circ}{R}$, whereas we have only assumed control over the first advective derivative $D_{t} \stackrel{\circ}{R}$. This difficulty is tackled in [18] by introducing a "mollification along the flow" for the definition of $\stackrel{\circ}{\ell}_{\ell}$, which is then used to form the amplitudes $a_{k}$ as in (36), (39). This procedure consists of first averaging in space to construct $\stackrel{\circ}{R}_{\ell_{x}}=\stackrel{\circ}{R} * \psi_{\ell_{x}}$ and then using the one-parameter group $\Xi_{s}(x, t)$ generated by the space-time vector field $\partial_{t}+v_{\ell} \cdot \nabla$ to average in time

$$
\stackrel{\circ}{R}_{\ell}(x, t)=\int \stackrel{\circ}{R}_{\ell_{x}}\left(\Xi_{s}(x, t)\right) \psi_{\ell_{t}}(s) d s .
$$

That is, $\Xi_{s}(x, t)=\left(X_{t}(x, t+s), t+s\right)$, where $X_{t}(x, t+s)$ denotes the flux of $v_{\ell}$ starting as the identity at time $t$, as in Appendix D. The tensor $\stackrel{\circ}{R}_{\ell}(x, t)$ is thus an average of $\stackrel{\circ}{R}$ over an $\ell_{x}$-neighborhood of the time $|s| \leq \ell_{t}$ trajectory that passes through $(x, t)$ along the flow of $v_{\ell}$.

The mollification (104) behaves like a standard mollification in that, because $D_{t}$ commutes with pullback by $\Xi_{s}$, the derivative $D_{t}$ commutes with the average along the flow

$$
\begin{aligned}
D_{t} \stackrel{\circ}{R}_{\ell}(x, t) & =\int\left[D_{t} \stackrel{\circ}{R}_{\ell_{x}}\right]\left(\Xi_{s}(x, t)\right) \psi_{\ell_{t}}(s) d s \\
& =\int \frac{d}{d s} \stackrel{\circ}{R}_{\ell_{x}}\left(\Xi_{s}(x, t)\right) \psi_{\ell_{t}}(s) d s .
\end{aligned}
$$

Integrating by parts in (105), each additional advective derivative incurs a cost $\ell_{t}^{-1}$, which provides the estimate on $D_{t}^{2} \stackrel{\circ}{R}_{\ell}$ that is applied to the transport term $D_{t} R^{0}$. The error $\stackrel{\circ}{R}-\stackrel{\circ}{R}_{\ell}$ can also be bounded in terms of $\ell_{t}$ and $\ell_{x}$ using

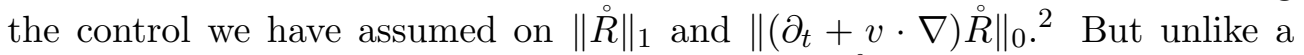
standard mollification, bounding the derivatives $\left\|\stackrel{\circ}{R}_{\ell}\right\|_{N}$ is more involved and requires control over the geometry of the flow $\Xi_{s}$ that obeys a nonlinear ODE. The bounds for $\left[\Xi_{s}\right]_{N}$ are then similar to those in Appendix D, having factors that grow exponentially after time $\|\nabla v\|_{0}^{-1}$ from Gronwall's inequality. After all these estimates are established, it turns out that the bounds for $D_{t}^{2} \stackrel{\circ}{R}_{\ell}$ and

\footnotetext{
${ }^{2}$ For the present results, one can essentially take $\ell_{t}$ proportional to the time scale $\mu^{-1}$ above, while obtaining a better exponent would require this parameter to be smaller (e.g., the choice made in [18] where $\ell_{t} \sim \delta_{q+1}^{-1 / 2} \lambda_{q+1}^{-1}$ would correspond to a $1 / 3$ scheme).
} 
$\stackrel{\circ}{R}-\stackrel{\circ}{R}_{\ell}$ all appear to be (just barely) compatible with a scheme aimed at proving the conjectured $1 / 3$ exponent. Overall though, it appears that $1 / 5$ is the limit of our current method and that any improvement on this exponent would require further new ideas.

\section{Conclusion of the proof}

In Sections $2-5$ we showed the construction for a single step, referring to $\left(v_{q}, p_{q}, \stackrel{\circ}{R}_{q}\right)$ as $(v, p, \stackrel{\circ}{R})$ and to $\left(v_{q+1}, p_{q+1}, \stackrel{\circ}{R}_{q+1}\right)$ as $\left(v_{1}, p_{1}, \stackrel{\circ}{R}_{1}\right)$. From now on we will consider the full iteration again, hence using again the indices $q$ and $q+1$.

In order to proceed, recall that the sequences $\left\{\delta_{q}\right\}_{q \in \mathbb{N}}$ and $\left\{\lambda_{q}\right\}_{q \in \mathbb{N}}$ are chosen to satisfy

$$
\delta_{q}=a^{-b^{q}}, \quad a^{c b^{q+1}} \leq \lambda_{q} \leq 2 a^{c b^{q+1}}
$$

for some given constants $c>5 / 2$ and $b>1$ and for $a>1$. Note that this has the consequence that if $a$ is chosen sufficiently large (depending only on $b>1$ ), then

$$
\delta_{q}^{1 / 2} \lambda_{q}^{1 / 5} \leq \delta_{q+1}^{1 / 2} \lambda_{q+1}^{1 / 5}, \quad \delta_{q+1} \leq \delta_{q}, \quad \text { and } \quad \lambda_{q} \leq \lambda_{q+1}^{\frac{2}{b+1}} .
$$

6.1. Choice of the parameters $\mu$ and $\ell$. We start by specifying the parameters $\mu=\mu_{q}$ and $\ell=\ell_{q}$ : we determine them optimizing the right-hand side of (93). More precisely, we set

$$
\mu:=\delta_{q+1}^{1 / 4} \delta_{q}^{1 / 4} \lambda_{q}^{1 / 2} \lambda_{q+1}^{1 / 2}
$$

so that the first two expressions in (93) are equal and then, having determined $\mu$, set

$$
\ell:=\delta_{q+1}^{-1 / 8} \delta_{q}^{1 / 8} \lambda_{q}^{-1 / 4} \lambda_{q+1}^{-3 / 4}
$$

so that the last two expressions in (93) are equal (up to a factor $\lambda_{q+1}^{\varepsilon}$ ).

In turn, these choices lead to

$$
\begin{aligned}
\left\|\stackrel{\circ}{R}_{q+1}\right\|_{0}+\frac{1}{\lambda_{q+1}}\left\|\stackrel{\circ}{R}_{q+1}\right\|_{1} & \leq C \delta_{q+1}^{3 / 4} \delta_{q}^{1 / 4} \lambda_{q}^{1 / 2} \lambda_{q+1}^{\varepsilon-1 / 2}+C \delta_{q+1}^{3 / 8} \delta_{q}^{5 / 8} \lambda_{q}^{3 / 4} \lambda_{q+1}^{\varepsilon-3 / 4} \\
& =C \delta_{q+1}^{3 / 4} \delta_{q}^{1 / 4} \lambda_{q}^{1 / 2} \lambda_{q+1}^{\varepsilon-1 / 2}\left(1+\left(\frac{\delta_{q}^{1 / 2} \lambda_{q}^{1 / 3}}{\delta_{q+1}^{1 / 2} \lambda_{q+1}^{1 / 3}}\right)^{3 / 4}\right) \\
& \stackrel{(106)}{\leq} C \delta_{q+1}^{3 / 4} \delta_{q}^{1 / 4} \lambda_{q}^{1 / 2} \lambda_{q+1}^{\varepsilon-1 / 2}
\end{aligned}
$$

Observe also that by (94), we have

$$
\left\|\partial_{t} \stackrel{\circ}{R}_{q+1}+v_{q+1} \cdot \nabla \stackrel{\circ}{R}_{q+1}\right\|_{0} \leq C \delta_{q+1}^{1 / 2} \lambda_{q+1}\left(\delta_{q+1}^{3 / 4} \delta_{q}^{1 / 4} \lambda_{q}^{1 / 2} \lambda_{q+1}^{\varepsilon-1 / 2}\right) .
$$


Let us check that the conditions (59) are satisfied for some $\beta>0$. (Remember that $\beta$ should be independent of $q$.) To this end we calculate

$$
\begin{aligned}
\frac{\delta_{q}^{1 / 2} \lambda_{q} \ell}{\delta_{q+1}^{1 / 2}}=\left(\frac{\delta_{q}^{1 / 2} \lambda_{q}^{3 / 5}}{\delta_{q+1}^{1 / 2} \lambda_{q+1}^{3 / 5}}\right)^{5 / 4}, & \frac{\delta_{q}^{1 / 2} \lambda_{q}}{\mu}=\left(\frac{\delta_{q}^{1 / 2} \lambda_{q}}{\delta_{q+1}^{1 / 2} \lambda_{q+1}}\right)^{1 / 2}, \\
\frac{1}{\ell \lambda_{q+1}}=\left(\frac{\delta_{q+1}^{1 / 2} \lambda_{q}}{\delta_{q}^{1 / 2} \lambda_{q+1}}\right)^{1 / 4}, & \frac{\mu}{\delta_{q+1}^{1 / 2} \lambda_{q+1}}=\left(\frac{\delta_{q}^{1 / 2} \lambda_{q}}{\delta_{q+1}^{1 / 2} \lambda_{q+1}}\right)^{1 / 2} .
\end{aligned}
$$

Hence the conditions (59) follow from (106) choosing $\beta=\frac{b-1}{5 b+5}$.

6.2. Proof of Proposition 0.2. Fix the constants $c>\frac{5}{2}$ and $b>1$ and also an $\varepsilon>0$ whose choice, like that of $a>1$, will be specified later. The proposition is proved inductively. The initial triple is defined to be $\left(v_{0}, p_{0}, \stackrel{\circ}{R}_{0}\right)=$ $(0,0,0)$. Now given $\left(v_{q}, p_{q}, \stackrel{\circ}{R}_{q}\right)$ satisfying the estimates (6)-(15), we claim that the triple $\left(v_{q+1}, p_{q+1}, \stackrel{\circ}{R+1}_{q}\right)$ constructed above satisfies again all the corresponding estimates.

Estimates on $\stackrel{\circ}{R}_{q+1}$. Note first of all that, using the form of the estimates in (93) and (94), the estimates (11) and (15) follow from (10). On the other hand, in light of (109), (10) follows from the recursion relation

$$
C \delta_{q+1}^{3 / 4} \delta_{q}^{1 / 4} \lambda_{q}^{1 / 2} \lambda_{q+1}^{\varepsilon-1 / 2} \leq \eta \delta_{q+2} .
$$

Using our choice of $\delta_{q}$ and $\lambda_{q}$ from Proposition 0.2, we see that this inequality is equivalent to

$$
C \leq a^{\frac{1}{4} b^{q}\left(1+3 b-2 c b+(2 c-4-4 \varepsilon c) b^{2}\right)}
$$

which, since $b>1$, is satisfied for all $q \geq 1$ for a sufficiently large fixed constant $a>1$, provided

$$
\left(1+3 b-2 c b+(2 c-4-4 \varepsilon c) b^{2}\right)>0 .
$$

Factorizing, we obtain the inequality $(b-1)((2 c-4) b-1)-4 \varepsilon c b^{2}>0$. It is then easy to see that for any $b>1$ and $c>5 / 2$, there exists $\varepsilon>0$ so that this inequality is satisfied. In this way we can choose $\varepsilon>0$ (and $\beta$ above) depending solely on $b$ and $c$. Consequently, this choice will determine all the constants in the estimates in Sections 2-5. We can then pick $a>1$ sufficiently large so that, by (109) and (110), the inequalities (10), (11) and (15) hold for $\stackrel{\circ}{R}_{q+1}$.

Estimates on $v_{q+1}-v_{q}$. By (50), Lemma 3.1 and (59), we conclude

$$
\begin{gathered}
\left\|v_{q+1}-v_{q}\right\|_{0} \leq\left\|w_{o}\right\|_{0}+\left\|w_{c}\right\|_{0} \leq \delta_{q+1}^{1 / 2}\left(\frac{M}{2}+\lambda_{q+1}^{-\beta}\right), \\
\left\|v_{q+1}-v_{q}\right\|_{1} \leq\left\|w_{o}\right\|_{1}+\left\|w_{c}\right\|_{1} \leq \delta_{q+1}^{1 / 2}\left(\frac{M}{2}+\lambda_{q+1}^{-\beta}\right) .
\end{gathered}
$$

Since $\lambda_{q+1} \geq \lambda_{1} \geq a^{c b^{2}}$, for $a$ sufficiently large we conclude (6) and (7). 
Estimate on the energy. Recall Lemma 4.1, and observe that, by (59), $\frac{\delta_{q+1}^{1 / 2} \delta_{q}^{1 / 2} \lambda_{q}}{\lambda_{q+1}} \leq \frac{\delta_{q+1}^{1 / 2} \mu}{\lambda_{q+1}}$. Moreover,

$$
\delta_{q+1} \delta_{q}^{1 / 2} \lambda_{q}=a^{-b^{q+1}-b^{q} / 2+c b^{q+1}}=a^{b^{q}((c-1) b-1 / 2)} \geq a \geq 1 .
$$

So the right-hand side of (80) is smaller than $C \frac{\delta_{q+1} \delta_{q}^{1 / 2} \lambda_{q}}{\mu}+C \frac{\delta_{q+1}^{1 / 2} \mu}{\lambda_{q+1}}$, i.e., smaller (up to a constant factor) than the right-hand side of (93). Thus, the argument used above to prove (10) also gives (12).

Estimates on $p_{q+1}-p_{1}$. From the definition of $p_{q+1}$ in (57) we deduce

$$
\left\|p_{q+1}-p_{q}\right\|_{0} \leq \frac{1}{2}\left(\left\|w_{o}\right\|_{0}+\left\|w_{c}\right\|_{0}\right)^{2}+C \ell\left\|v_{q}\right\|_{1}\|w\|_{0} .
$$

As already argued in the estimate for (6), $\left\|w_{o}\right\|+\left\|w_{c}\right\| \leq M \delta_{q}^{1 / 2}$. Moreover, $C \ell\left\|v_{1}\right\|_{1}\|w\|_{0} \leq C M \delta_{q+1}^{1 / 2} \delta_{q}^{1 / 2} \lambda_{q} \ell$, which is smaller than the right-hand side of (93). Having already argued that such quantity is smaller than $\eta \delta_{q+2}$, we can obviously bound $C \ell\left\|v_{q}\right\|_{1}\|w\|_{0}$ with $\frac{M^{2}}{2} \delta_{q+1}$. This shows (8). Moreover, differentiating (57), we achieve the bound

$$
\left\|p_{q+1}-p_{q}\right\|_{1} \leq\left(\left\|w_{o}\right\|_{1}+\left\|w_{c}\right\|_{1}\right)\left(\left\|w_{o}\right\|_{0}+\left\|w_{c}\right\|_{0}\right)+C \delta_{q+1}^{1 / 2} \delta_{q}^{1 / 2} \lambda_{q} \lambda_{q+1} \ell,
$$

and arguing as above we conclude (9).

Estimates (16). Here we can use the obvious identity $\partial_{t} w_{q}=D_{t} w_{q}-\left(v_{q}\right)_{\ell}$. $\nabla w_{q}$ together with Lemmas 3.1 and 3.2 to obtain $\left\|\partial_{t} v_{q+1}-\partial_{t} v_{q}\right\|_{0} \leq C \delta_{q+1}^{1 / 2} \lambda_{q+1}$. Then, using (25), we conclude $\left\|\partial_{t} v_{q}\right\|_{0} \leq C \delta_{q}^{1 / 2} \lambda_{q}$.

To handle $\partial_{t} p_{q+1}-\partial_{t} p_{q}$, observe first that, by our construction,

$$
\begin{aligned}
\left\|\partial_{t}\left(p_{q+1}-p_{q}\right)\right\|_{0} \leq & \left(\left\|w_{c}\right\|_{0}+\left\|w_{o}\right\|_{0}\right)\left(\left\|\partial_{t} w_{c}\right\|_{0}+\left\|\partial_{t} w_{o}\right\|_{0}\right) \\
& +2\|w\|_{0}\left\|\partial_{t} v_{q}\right\|_{0}+\ell\left\|v_{q}\right\|_{1}\left\|\partial_{t} w\right\|_{0} .
\end{aligned}
$$

As above, we can derive the estimates $\left\|\partial_{t} w_{o}\right\|_{0}+\left\|\partial_{t} w_{c}\right\|_{0} \leq C \delta_{q+1}^{1 / 2} \lambda_{q+1}$ from Lemmas 3.1 and 3.2. Hence

$$
\left\|\partial_{t}\left(p_{q+1}-p_{q}\right)\right\|_{0} \leq C \delta_{q+1} \lambda_{q+1}+C \delta_{q+1}^{1 / 2} \delta_{q}^{1 / 2} \lambda_{q}+C \delta_{q}^{1 / 2} \lambda_{q} \ell \delta_{q+1}^{1 / 2} \lambda_{q+1} .
$$

Since $\ell \leq \lambda_{q}^{-1}$ and $\delta_{q}^{1 / 2} \lambda_{q} \leq \delta_{q+1}^{1 / 2} \lambda_{q+1}$, the desired inequality follows. This concludes the proof.

\section{Onsager's conjecture almost everywhere in time}

In recent work [1] by the first author, a variant of the present scheme was presented in order to prove the existence of nontrivial Euler flows with compact temporal support that belong to the Hölder class $C^{1 / 3-\varepsilon}$ almost everywhere in time. Specifically, the following theorem was proved. 
THEOREM 7.1. There exists a nontrivial vector field

$$
v \in C^{1 / 5-\varepsilon}\left(\mathbb{T}^{3} \times(-1,1), \mathbb{R}^{3}\right)
$$

with compact support in time and a scalar field

$$
p \in C^{2 / 5-2 \varepsilon}\left(\mathbb{T}^{3} \times(-1,1)\right)
$$

with the following properties:

(i) The pair $(v, p)$ solves the incompressible Euler equations (1) in the sense of distributions.

(ii) There exists a set $\Omega \subset(-1,1)$ of Hausdorff dimension strictly less than 1 such that if $t \notin \Omega$, then $v(\cdot, t)$ is Hölder $C^{1 / 3-\varepsilon}$ continuous and $p$ is Hölder $C^{2 / 3-2 \varepsilon}$ continuous. ${ }^{3}$

The construction of the convex iteration scheme in [1] follows very closely the scheme presented here with two main deviations:

(1) Unlike in the present work, the solutions constructed will not obey any prescribed energy profile, but rather simply satisfy the property of having compact support in time. ${ }^{4}$

(2) The time cutoff functions $\left\{\chi_{q, l}\right\}^{5}$ are modified in such a way that the Hausdorff dimension of the set

$$
\Omega^{\prime}:=\bigcap_{q=1}^{\infty} \bigcup_{q^{\prime}=q}^{\infty} \bigcup_{l} \operatorname{support}\left(\chi_{q^{\prime}, l}^{\prime}\right)
$$

is strictly less than 1 .

The proof of the above theorem then relies heavily on the estimates given in the present work with the addition of sharper, time localized estimates. The set $\Omega$ is taken to be a slight enlargement of $\Omega^{\prime}$ with identical Hausdorff dimension. With the help of these additional estimates, it is shown that for any time $t \in(-1,1)$ outside the set $\Omega$, there exists an $N=N(t)$ such that, suppressing dependence on $t$, we obtain the following estimates:

$$
\begin{array}{r}
\left\|w_{q}\right\|_{0}+\frac{1}{\lambda_{q}}\left\|\partial_{t} w_{q}\right\|_{0}+\frac{1}{\lambda_{q}}\left\|w_{q}\right\|_{1} \leq \lambda_{q}^{-1 / 3+\varepsilon_{0}}, \\
\left\|p_{q}-p_{q-1}\right\|_{0}+\frac{1}{\lambda_{q}}\left\|\partial_{t}\left(p_{q}-p_{q-1}\right)\right\|_{0}+\frac{1}{\lambda_{q}^{2}}\left\|p_{q}-p_{q-1}\right\|_{2} \leq \lambda_{q}^{-2 / 3+2 \varepsilon_{0}}, \\
\left\|\stackrel{\circ}{R}_{q}\right\|_{0}+\frac{1}{\lambda_{q}}\left\|\stackrel{\circ}{R}_{q}\right\|_{1} \leq \lambda_{q+1}^{-2 / 3+2 \varepsilon_{0}}
\end{array}
$$

\footnotetext{
${ }^{3}$ More precisely, the Hausdorff dimension $d$ is such that $1-d>C \varepsilon^{2}$ for some positive constant $C$.

${ }^{4}$ This approach is also taken in [18].

${ }^{5}$ Here we include the subscript $q$ to indicate the iteration in which the cutoff is defined.
} 
for every $q \geq N$ and some $0<\varepsilon_{0}<\varepsilon$. The ability to obtain such estimates is intimately related to the property $\Omega^{\prime} \subset \Omega$.

A minor detail to note is that unlike in the present scheme, in [1] one must keep track of second order estimates on the pressure (as was already observed in [18]; cf. Remark 3). This is done in order to eliminate the second term appearing in (87), whose appearance is related to the sub-optimal estimates (74) and (76). While this term poses no problems in the present work, it seems to unduly provide restrictions on the mollification parameter $\ell$, which in turn provides an obstruction to proving Theorem 7.1.

\section{Appendix A. Proof of Proposition 1.1}

Proof. First of all observe that $a_{-k} B_{-k}=\overline{a_{k} B_{k}}$. Thus the vector field defined in (19) is real valued. Next notice that

$$
\operatorname{div} W(\xi)=\sum_{|k|=\lambda_{0}} i k \cdot B_{k} a_{k} e^{i k \cdot \xi}=0,
$$

because $k \cdot B_{k}=0$ for every $k$.

In turn we directly compute

$$
\begin{aligned}
\operatorname{div}(W \otimes W)(\xi) & =\sum_{k, k^{\prime}} i\left(B_{k} \otimes B_{k}^{\prime}\right) \cdot\left(k+k^{\prime}\right) e^{i\left(k+k^{\prime}\right) \cdot \xi} \\
& =\sum_{k, k^{\prime}} \frac{i}{2}\left(B_{k} \otimes B_{k^{\prime}}+B_{k^{\prime}} \otimes B_{k}\right) \cdot\left(k+k^{\prime}\right) e^{i\left(k+k^{\prime}\right) \cdot \xi} \\
& \stackrel{(22)}{=} \sum_{k, k^{\prime}}\left(B_{k} \cdot B_{k}^{\prime}\right)\left(k+k^{\prime}\right) e^{i\left(k+k^{\prime}\right) \cdot \xi}=\nabla \frac{1}{2} \sum_{k, k^{\prime}} B_{k} \cdot B_{k}^{\prime} e^{i\left(k+k^{\prime}\right) \cdot \xi} \\
& =\frac{1}{2} \nabla(W \cdot W)=\frac{1}{2} \nabla(W \cdot \bar{W})=\frac{1}{2} \nabla|W|^{2} .
\end{aligned}
$$

Averaging this identity in $\xi$ we infer

$$
\langle W \otimes W\rangle=\sum_{|k|=\lambda_{0}}\left|a_{k}\right|^{2} B_{k} \otimes \bar{B}_{k} .
$$

However, since $B_{k}=\bar{B}_{-k}$, we get

$$
\begin{aligned}
\langle W \otimes W\rangle & =\sum_{|k|=\lambda_{0}}\left|a_{k}\right|^{2} \operatorname{Re}\left(B_{k} \otimes \bar{B}_{k}\right) \\
& =\sum_{|k|=\lambda_{0}}\left|a_{k}\right|^{2}\left(A_{k} \otimes A_{k}+\left(\frac{k}{|k|} \times A_{k}\right) \otimes\left(\frac{k}{|k|} \times A_{k}\right)\right) .
\end{aligned}
$$


On the other hand, observe that the triple $\sqrt{2} A_{k}, \sqrt{2} \frac{k}{|k|} \times A_{k}, \frac{k}{|k|}$ forms an orthonormal basis of $\mathbb{R}^{3}$. Thus,

$$
2 A_{k} \otimes A_{k}+2\left(\frac{k}{|k|} \times A_{k}\right) \otimes\left(\frac{k}{|k|} \times A_{k}\right)+\frac{k}{|k|} \otimes \frac{k}{|k|}=\mathrm{Id} .
$$

This shows (21) and hence completes the proof.

\section{Appendix B. Proof of Lemma 1.3}

The lemma was first proved in [11], where the argument is very close to the classical decomposition of a Riemannian metric into "primitive ones," a fundamental point of Nash's existence theorem for $C^{1}$-isometric embeddings; see [20] and also [17]. The proof reported here is instead closer in spirit to [18] and gives more explicit formulas for the families of vectors $\Lambda_{j}$.

In this paper the lemma is applied with $N=2$ (compare with [11] and [7] where it is applied with $N=8$ ). However, the general form might be useful in different contexts. For instance, in order to handle a general torus $\mathbb{R}^{3} / \Gamma$, a larger $N$ and a suitable variant of the lemma seem necessary; see [18] for the details.

Proof. The case $N=1$. Let $e_{1}, e_{2}, e_{3}$ be the standard basis vectors of $\mathbb{R}^{3}$, and define the sets

$$
\Lambda_{1}=\left\{ \pm\left(e_{i} \pm e_{j}\right) \mid 1 \leq i<j \leq 3\right\} \subseteq \mathbb{Z}^{3} \cap\{|k|=\sqrt{2}\}
$$

and

$$
\Lambda_{1}^{+}=\left\{\left(e_{i} \pm e_{j}\right) \mid 1 \leq i<j \leq 3\right\},
$$

which are integral analogues of the dodecahedron and projective dodecahedron used in [18]. Note that $\Lambda_{1}$ is invariant under the finite reflection group $G$ generated by the permutations of the basis vectors $\left(e_{1}, e_{2}, e_{3}\right)$ together with the reflections about coordinate planes and coordinate axes, which negate one or two basis vectors respectively. We have the following properties.

(1) Linear Independence. The tensors

$$
\mathcal{B}=\left\{\operatorname{Id}-\frac{k \otimes k}{|k|^{2}} \mid k \in \Lambda_{1}^{+}\right\}
$$

are linearly independent and hence form a basis for the space of symmetric matrices, here viewed as bilinear forms.

(2) Symmetry. We have the identity

$$
\frac{1}{2} \sum_{k \in \Lambda_{1}}\left(\mathrm{Id}-\frac{k \otimes k}{|k|^{2}}\right)=4 \cdot \mathrm{Id} .
$$


To establish (119), consider a linear dependence relation

$$
\sum_{k \in \Lambda_{1}^{+}} C_{k}\left(\mathrm{Id}-\frac{k \otimes k}{|k|^{2}}\right)=0
$$

The space of linear relations of the form (121) gives a linear representation of the group $G$, which arises from the action of $G$ on the set $\mathcal{B}$. Since the action of $G$ is transitive, it suffices to show that $C_{e_{1}+e_{2}}=0$, which we now prove.

After averaging (121) over the subgroup permuting the set $\left\{ \pm\left(e_{1} \pm e_{2}\right)\right\}$ (which acts transitively on $\Lambda_{1} \backslash\left\{ \pm\left(e_{1} \pm e_{2}\right)\right\}$ ), we can arrange that all the coefficients $C_{\left(e_{i} \pm e_{3}\right)}=\hat{C}$ are equal for $i=1,2$, and from (121), we obtain

$$
\sum_{ \pm} C_{\left(e_{1} \pm e_{2}\right)}\left(\operatorname{Id}-\frac{\left(e_{1} \pm e_{2}\right)^{2}}{\left|e_{1} \pm e_{2}\right|^{2}}\right)=-\hat{C} \sum_{k \in \Lambda_{1}^{+} \backslash\left\{e_{1} \pm e_{2}\right\}}\left(\operatorname{Id}-\frac{k \otimes k}{|k|^{2}}\right)
$$

Comparing the equations obtained from taking traces versus applying (122) to $e_{3} \otimes e_{3}$, we conclude that $\hat{C}=0$. Then applying (122) to $\left(e_{1} \pm e_{2}\right) \otimes\left(e_{1} \pm e_{2}\right)$, we conclude that $C_{e_{1} \pm e_{2}}=0$ as desired.

The identity (120) follows from the linear independence of $\mathcal{B}$, which implies that there are unique coefficients $C_{k}$ solving

$$
\sum_{k \in \Lambda_{1}^{+}} C_{k}\left(\operatorname{Id}-\frac{k \otimes k}{|k|^{2}}\right)=\mathrm{Id} .
$$

By uniqueness, the coefficients $C_{k}$ must all be equal upon averaging over $G$. Equation (120) then follows by comparing traces.

Lemma 1.3 for $N=1$ is now an immediate corollary of the properties (119) and (120). Namely, as long as $\varepsilon$ varies among symmetric matrices in some ball $B_{r_{0}}(0)$, the equation

$$
\frac{1}{2} \sum_{k \in \Lambda_{1}}\left(\gamma_{k}^{(1)}\right)^{2}\left(\mathrm{Id}-\frac{k \otimes k}{|k|^{2}}\right)=\mathrm{Id}+\varepsilon
$$

has unique, positive solutions $\gamma_{k}^{(1)}$ for which $\gamma_{-k}^{(1)}=\gamma_{k}^{(1)}$ and

$$
\gamma_{k}^{(1)}(\operatorname{Id}+\varepsilon)-\frac{1}{2}=O(|\varepsilon|)
$$

The smooth dependence of $\gamma_{k}^{(1)}$ on $\varepsilon$ in the ball $B_{r_{0}}(0)$ is also clear.

Remark 4. One can also see equation (120) from the fact that Id is, up to a constant, the unique, symmetric bilinear form invariant under the action 
of $G{ }^{6}$ This approach is closer to the proof in [18], which uses the identity (120) for the dodecahedron to prove the linear independence (119) through a symmetry argument.

The case $N>1$. Now assume $N>1$, and let $\Lambda^{*}$ be the family of integer vectors constructed from the case $N=1$. It suffices to obtain families of integer vectors $\Lambda_{j}, j=1,2, \ldots, N$ that all lie on the same sphere and have the properties (119) and (120). This can be done as follows. By rotating the family $\Lambda^{*}$, we can easily produce arbitrarily many families of vectors $\Lambda_{j}^{\mathbb{R}} \subseteq$ $\mathbb{R}^{3} \cap\{|k|=\sqrt{2}\}, j=1, \ldots N$ that are disjoint from each other and for which the properties of linear independence (119) and symmetry (120) also hold. For example, we can use families of the form

$$
\Lambda_{j}^{\mathbb{R}}=\left\{e^{\theta_{j} e_{1} \times} k \mid k \in \Lambda^{*}\right\}
$$

that are obtained from the one-parameter group of rotations

$$
e^{\theta e_{1} \times} k=\left[\begin{array}{ccc}
1 & 0 & 0 \\
0 & \cos \theta & -\sin \theta \\
0 & \sin \theta & \cos \theta
\end{array}\right] k
$$

that rotate $\Lambda^{*}$ about the $e_{1}$ axis without leaving any member of $\Lambda^{*}$ fixed. For generic choices of $\theta_{j} \in \mathbb{R} /(2 \pi \mathbb{Z}), j=1, \ldots, N$, the families $\Lambda_{j}^{\mathbb{R}}$ will be pairwise disjoint but will not contain vectors in $\mathbb{Z}^{3}$. However, for a dense subset of $\theta_{j}$, the values $\left(\cos \theta_{j}, \sin \theta_{j}\right)$ will be rational numbers, which can be seen, for instance, by mapping $\mathbb{Q} \subseteq \mathbb{R}$ into a dense subset of the circle by the inverse of the stereographic projection

$$
x \mapsto\left(\frac{2 x}{1+x^{2}}, \frac{x^{2}-1}{1+x^{2}}\right) .
$$

Restricting to these choices of $\theta_{j}$, we obtain arbitrarily many families

$$
\Lambda_{j}^{\mathbb{Q}}=\left\{e^{\theta_{j} e_{1} \times} k \mid k \in \Lambda^{*}\right\} \subseteq \mathbb{Q}^{3} \cap\{|k|=\sqrt{2}\} \quad j=1, \ldots, N
$$

that are pairwise disjoint and have rational entries. Having chosen $N$ such $\Lambda_{j}^{\mathbb{Q}}$, we obtain the desired $\Lambda_{1}, \ldots, \Lambda_{N}$ by scaling $\Lambda_{j}=M \Lambda_{j}^{\mathbb{Q}}$ by an integer $M$ chosen to ensure $\Lambda_{j} \subseteq \mathbb{Z}^{3} \cap\{|k|=\sqrt{2} M\}$. As properties (119) and (120) are not disturbed by rotation and scaling, this proves Lemma 1.3 with $\lambda=\sqrt{2} M$ and $r_{0}$ independent of $N$.

\footnotetext{
${ }^{6}$ One calculates the dimension of the space of $G$-invariant symmetric bilinear forms by taking the trace of the projection operator $\frac{1}{|G|} \sum_{g \in G} \mathrm{Sym}^{2} g$ and using the identity $\operatorname{tr} \operatorname{Sym}^{2} g=\left(\operatorname{tr} g^{2}+(\operatorname{tr} g)^{2}\right) / 2$.
} 


\section{Appendix C. Hölder spaces}

In the following, $m=0,1,2, \ldots, \alpha \in(0,1)$ and $\beta$ is a multi-index. We introduce the usual (spatial) Hölder norms as follows. First of all, the supremum norm is denoted by $\|f\|_{0}:=\sup _{\mathbb{T}^{3} \times[0,1]}|f|$. We define the Hölder seminorms as

$$
\begin{aligned}
{[f]_{m} } & =\max _{|\beta|=m}\left\|D^{\beta} f\right\|_{0}, \\
{[f]_{m+\alpha} } & =\max _{|\beta|=m} \sup _{x \neq y, t} \frac{\left|D^{\beta} f(x, t)-D^{\beta} f(y, t)\right|}{|x-y|^{\alpha}}
\end{aligned}
$$

where $D^{\beta}$ are space derivatives only. The Hölder norms are then given by

$$
\begin{aligned}
\|f\|_{m} & =\sum_{j=0}^{m}[f]_{j}, \\
\|f\|_{m+\alpha} & =\|f\|_{m}+[f]_{m+\alpha} .
\end{aligned}
$$

Moreover, we will write $[f(t)]_{\alpha}$ and $\|f(t)\|_{\alpha}$ when the time $t$ is fixed and the norms are computed for the restriction of $f$ to the $t$-time slice.

Recall the following elementary inequalities:

$$
[f]_{s} \leq C\left(\varepsilon^{r-s}[f]_{r}+\varepsilon^{-s}\|f\|_{0}\right)
$$

for $r \geq s \geq 0, \varepsilon>0$ and

$$
[f g]_{r} \leq C\left([f]_{r}\|g\|_{0}+\|f\|_{0}[g]_{r}\right)
$$

for any $1 \geq r \geq 0$. From (126) with $\varepsilon=\|f\|_{0}^{\frac{1}{r}}[f]_{r}^{-\frac{1}{r}}$ we obtain the standard interpolation inequalities

$$
[f]_{s} \leq C\|f\|_{0}^{1-\frac{s}{r}}[f]_{r}^{\frac{s}{r}}
$$

Next we collect two classical estimates on the Hölder norms of compositions. These are also standard, for instance, in applications of the Nash-Moser iteration technique.

Proposition C.1. Let $\Psi: \Omega \rightarrow \mathbb{R}$ and $u: \mathbb{R}^{n} \rightarrow \Omega$ be two smooth functions, with $\Omega \subset \mathbb{R}^{N}$. Then, for every $m \in \mathbb{N} \backslash\{0\}$, there is a constant $C$ (depending only on $m, N, n$ ) such that

$$
\begin{aligned}
& {[\Psi \circ u]_{m} \leq C\left([\Psi]_{1}[u]_{m}+\|D \Psi\|_{m-1}\|u\|_{0}^{m-1}[u]_{m}\right),} \\
& {[\Psi \circ u]_{m} \leq C\left([\Psi]_{1}[u]_{m}+\|D \Psi\|_{m-1}[u]_{1}^{m}\right) .}
\end{aligned}
$$




\section{Appendix D. Estimates for transport equations}

In this section we recall some well-known results regarding smooth solutions of the transport equation:

$$
\left\{\begin{array}{l}
\partial_{t} f+v \cdot \nabla f=g \\
\left.f\right|_{t_{0}}=f_{0}
\end{array}\right.
$$

where $v=v(t, x)$ is a given smooth vector field. We denote the advective derivative $\partial_{t}+v \cdot \nabla$ by $D_{t}$. We will consider solutions on the entire space $\mathbb{R}^{3}$ and treat solutions on the torus simply as periodic solution in $\mathbb{R}^{3}$.

Proposition D.1. Assume $t>t_{0}$. Any solution $f$ of (131) satisfies

$$
\begin{aligned}
\|f(t)\|_{0} & \leq\left\|f_{0}\right\|_{0}+\int_{t_{0}}^{t}\|g(\tau)\|_{0} d \tau \\
{[f(t)]_{1} } & \leq\left[f_{0}\right]_{1} e^{\left(t-t_{0}\right)[v]_{1}}+\int_{t_{0}}^{t} e^{(t-\tau)[v]_{1}}[g(\tau)]_{1} d \tau
\end{aligned}
$$

and, more generally, for any $N \geq 2$, there exists a constant $C=C_{N}$ so that

$$
\begin{aligned}
{[f(t)]_{N} \leq } & \left(\left[f_{0}\right]_{N}+C\left(t-t_{0}\right)[v]_{N}\left[f_{0}\right]_{1}\right) e^{C\left(t-t_{0}\right)[v]_{1}} \\
& +\int_{t_{0}}^{t} e^{C(t-\tau)[v]_{1}}\left([g(\tau)]_{N}+C(t-\tau)[v]_{N}[g(\tau)]_{1}\right) d \tau .
\end{aligned}
$$

Define $\Phi(t, \cdot)$ to be the inverse of the flux $X$ of $v$ starting at time $t_{0}$ as the identity (i.e., $\frac{d}{d t} X=v(X, t)$ and $\left.X\left(x, t_{0}\right)=x\right)$. Under the same assumptions as above,

$$
\begin{aligned}
\|D \Phi(t)-\mathrm{Id}\|_{0} & \leq e^{\left(t-t_{0}\right)[v]_{1}}-1, \\
{[\Phi(t)]_{N} } & \leq C\left(t-t_{0}\right)[v]_{N} e^{C\left(t-t_{0}\right)[v]_{1}} \quad \forall N \geq 2 .
\end{aligned}
$$

Proof. We start with the following elementary observation for transport equations: if $f$ solves (131), then $\frac{d}{d t} f(X(t, x), t)=g(X(t, x), t)$ and consequently

$$
f(t, x)=f_{0}(\Phi(x, t))+\int_{t_{0}}^{t} g(X(\Phi(t, x), \tau), \tau) d \tau .
$$

The maximum principle (132) follows immediately. Next, differentiate (131) in $x$ to obtain the identity

$$
D_{t} D f=\left(\partial_{t}+v \cdot \nabla\right) D f=D g-D f D v .
$$

Applying (132) to $D f$ yields

$$
[f(t)]_{1} \leq\left[f_{0}\right]_{1}+\int_{t_{0}}^{t}\left([g(\tau)]_{1}+[v]_{1}[f(\tau)]_{1}\right) d \tau .
$$

An application of Gronwall's inequality then yields (133). 
More generally, differentiating (131) $N$ times yields

$$
\partial_{t} D^{N} f+(v \cdot \nabla) D^{N} f=D^{N} g+\sum_{j=0}^{N-1} c_{j, N} D^{j+1} f: D^{N-j} v
$$

(where : is a shorthand notation for sums of products of entries of the corresponding tensors).

Also, using (132) and the interpolation inequality (128), we can estimate

$$
[f(t)]_{N} \leq\left[f_{0}\right]_{N}+\int_{t_{0}}^{t}\left([g(\tau)]_{N}+C\left([v]_{N}[f(\tau)]_{1}+[v]_{1}[f(\tau)]_{N}\right)\right) d \tau .
$$

Now plugging the estimate (133), Gronwall's inequality leads — after some elementary calculations - to (134).

The estimate (136) follows easily from (134) observing that $\Phi$ solves (131) with $g=0$ and $D^{2} \Phi\left(\cdot, t_{0}\right)=0$. Next consider $\Psi(x, t)=\Phi(x, t)-x$. First observe that $\partial_{t} \Psi+v \cdot \nabla \Psi=-v$. Since $D \Psi\left(\cdot, t_{0}\right)=0$, we apply (133) to conclude

$$
[\Psi(t)]_{1} \leq \int_{t_{0}}^{t} e^{(t-\tau)[v]_{1}}[v]_{1} d \tau=e^{\left(t-t_{0}\right)[v]_{1}}-1 .
$$

Since $D \Psi(x, t)=D \Phi(x, t)-\mathrm{Id},(135)$ follows.

\section{Appendix E. Constantin-E-Titi commutator estimate}

Finally, we recall the quadratic commutator estimate from [3] (also cf. [4, Lemma 1]):

Proposition E.1. Let $f, g \in C^{\infty}\left(\mathbb{T}^{3} \times \mathbb{T}\right)$ and $\psi$ the mollifier of Section 2. For any $r \geq 0$, we have the estimate

$$
\left\|\left(f * \psi_{\ell}\right)\left(g * \psi_{\ell}\right)-(f g) * \psi_{\ell}\right\|_{r} \leq C \ell^{2-r}\|f\|_{1}\|g\|_{1},
$$

where the constant $C$ depends only on $r$.

\section{Appendix F. Schauder estimates}

Here we recall the following consequences of the classical Schauder estimates (cf. [11, Prop. 5.1]).

Proposition F.1. For any $\alpha \in(0,1)$ and any $m \in \mathbb{N}$, there exists a constant $C(\alpha, m)$ with the following properties. If $\phi, \psi: \mathbb{T}^{3} \rightarrow \mathbb{R}$ are the unique solutions of

$$
\left\{\begin{array} { l } 
{ \Delta \phi = f , } \\
{ f \phi = 0 }
\end{array} \quad \text { and } \quad \left\{\begin{array}{l}
\Delta \psi=\operatorname{div} F, \\
f \psi=0
\end{array}\right.\right.
$$

then

$$
\|\phi\|_{m+2+\alpha} \leq C(m, \alpha)\|f\|_{m, \alpha} \quad \text { and } \quad\|\psi\|_{m+1+\alpha} \leq C(m, \alpha)\|F\|_{m, \alpha} .
$$


Moreover, we have the estimates

$$
\begin{aligned}
\|\mathcal{R} v\|_{m+1+\alpha} & \leq C(m, \alpha)\|v\|_{m+\alpha} \\
\|\mathcal{R}(\operatorname{div} A)\|_{m+\alpha} & \leq C(m, \alpha)\|A\|_{m+\alpha} .
\end{aligned}
$$

Proof. The estimates (139) and (140) are easy consequences of (138) and the definition of the $\mathcal{R}$ operator. The estimates (138) are the usual Schauder estimates; see, for instance, [16, Chap. 4]. The meticulous reader will notice that the estimates in [16] are stated in $\mathbb{R}^{n}$ for the potential-theoretic solution of the Laplace operator. The periodic case is, however, an easy corollary. (See [11] for the details.)

\section{Appendix G. Stationary phase lemma}

We recall the following simple facts here. For completeness, we include the proof given in [11].

Proposition G.1.

(i) Let $k \in \mathbb{Z}^{3} \backslash\{0\}$ and $\lambda \geq 1$ be fixed. For any $a \in C^{\infty}\left(\mathbb{T}^{3}\right)$ and $m \in \mathbb{N}$, we have

$$
\left|\int_{\mathbb{T}^{3}} a(x) e^{i \lambda k \cdot x} d x\right| \leq \frac{[a]_{m}}{\lambda^{m}} .
$$

(ii) Let $k \in \mathbb{Z}^{3} \backslash\{0\}$ be fixed. For a smooth vector field $a \in C^{\infty}\left(\mathbb{T}^{3} ; \mathbb{R}^{3}\right)$, let $F(x):=a(x) e^{i \lambda k \cdot x}$. Then we have

$$
\|\mathcal{R}(F)\|_{\alpha} \leq \frac{C}{\lambda^{1-\alpha}}\|a\|_{0}+\frac{C}{\lambda^{m-\alpha}}[a]_{m}+\frac{C}{\lambda^{m}}[a]_{m+\alpha},
$$

where $C=C(\alpha, m)$.

Proof. For $j=0,1, \ldots$, define

$$
\begin{aligned}
& A_{j}(y, \xi):=-i\left[\frac{k}{|k|^{2}}\left(i \frac{k}{|k|^{2}} \cdot \nabla\right)^{j} a(y)\right] e^{i k \cdot \xi}, \\
& F_{j}(y, \xi):=\left[\left(i \frac{k}{|k|^{2}} \cdot \nabla\right)^{j} a(y)\right] e^{i k \cdot \xi} .
\end{aligned}
$$

Direct calculation shows that

$$
F_{j}(x, \lambda x)=\frac{1}{\lambda} \operatorname{div}\left[A_{j}(x, \lambda x)\right]+\frac{1}{\lambda} F_{j+1}(x, \lambda x) .
$$

In particular, for any $m \in \mathbb{N}$,

$$
a(x) e^{i \lambda k \cdot x}=F_{0}(x, \lambda x)=\frac{1}{\lambda} \sum_{j=0}^{m-1} \frac{1}{\lambda^{j}} \operatorname{div}\left[A_{j}(x, \lambda x)\right]+\frac{1}{\lambda^{m}} F_{m}(x, \lambda x) .
$$

Integrating this over $\mathbb{T}^{3}$ and using that $|k| \geq 1$, we obtain (141). 
Consider next the solution $\phi_{\lambda} \in C^{\infty}\left(\mathbb{T}^{3}\right)$ of

$$
\Delta \phi_{\lambda}=f_{\lambda} \text { in } \mathbb{T}^{3}
$$

with $\int_{\mathbb{T}^{3}} \phi_{\lambda}=0$, where

$$
f_{\lambda}(x):=a(x) e^{i \lambda k \cdot x}-f_{\mathbb{T}^{3}} a(y) e^{i \lambda k \cdot y} d y .
$$

Then for any $\alpha \in(0,1)$ and $m \in \mathbb{N}$, we claim the estimate

$$
\left\|\nabla \phi_{\lambda}\right\|_{\alpha} \leq \frac{C}{\lambda^{1-\alpha}}\|a\|_{0}+\frac{C}{\lambda^{m-\alpha}}[a]_{m}+\frac{C}{\lambda^{m}}[a]_{m+\alpha},
$$

where $C=C(\alpha, m)$.

Indeed, using (126) and (127) with the $F_{j}$ 's and $A_{j}$ 's introduced above, we have for any $j \leq m-1$,

$$
\begin{aligned}
\left\|A_{j}(\cdot, \lambda \cdot)\right\|_{\alpha} & \leq C\left(\lambda^{\alpha}[a]_{j}+[a]_{j+\alpha}\right) \\
& \leq C \lambda^{j+\alpha}\left(\lambda^{-m}[a]_{m}+\|a\|_{0}\right)
\end{aligned}
$$

and similarly,

$$
\left\|F_{m}(\cdot, \lambda \cdot)\right\|_{\alpha} \leq C\left(\lambda^{\alpha}[a]_{m}+[a]_{m+\alpha}\right) .
$$

Moreover, according to the standard estimate (138),

$$
\|\nabla \phi\|_{\alpha} \leq C\left(\frac{1}{\lambda} \sum_{j=0}^{m-1} \frac{1}{\lambda^{j}}\left\|A_{j}(\cdot, \lambda \cdot)\right\|_{\alpha}+\frac{1}{\lambda^{m}}\left\|F_{m}(\cdot, \lambda \cdot)\right\|_{\alpha}+\left|f_{\mathbb{T}^{3}} F_{0}(x, \lambda x) d x\right|\right),
$$

hence, using (141) for the last term,

$$
\|\nabla \phi\|_{\alpha} \leq \frac{C}{\lambda^{1-\alpha}}\|a\|_{0}+\frac{C}{\lambda^{m-\alpha}}[a]_{m}+\frac{C}{\lambda^{m}}[a]_{m+\alpha}
$$

as required.

Taking into account the definition of $\mathcal{R}$, the estimate (142) follows now applying (143) and Proposition F.1 above. (Recall also that, if $v$ is a vector field, its Leray projection $\mathcal{P}(v)$ is given by $v-f v-\nabla \phi$, where $\Delta \phi=\operatorname{div} v$.)

\section{Appendix H. One further commutator estimate}

Proposition H.1. Let $k \in \mathbb{Z}^{3} \backslash\{0\}$ be fixed. For any smooth vector field $a \in C^{\infty}\left(\mathbb{T}^{3} ; \mathbb{R}^{3}\right)$ and any smooth function $b$, if we set $F(x):=a(x) e^{i \lambda k \cdot x}$, we then have

$$
\|[b, \mathcal{R}](F)\|_{\alpha} \leq \lambda^{\alpha-2}\|a\|_{0}\|b\|_{1}+C \lambda^{\alpha-m}\left(\|a\|_{m-1+\alpha}\|b\|_{1+\alpha}+\|a\|_{\alpha}\|b\|_{m+\alpha}\right),
$$

where $C=C(\alpha, m)$.

Proof. Step 1. First of all, given a vector field $v$, define the operator

First observe that

$$
\mathcal{S}(v):=\nabla v+(\nabla v)^{t}-\frac{2}{3}(\operatorname{div} v) \mathrm{Id} .
$$

$$
\operatorname{div} \mathcal{S}(v)=0 \quad \Longleftrightarrow \quad v \equiv \text { const. }
$$


One implication is obvious. Next, assume $\operatorname{div} \mathcal{S}(v)=0$. This is equivalent to the equations

$$
\Delta v_{j}+\frac{1}{3} \partial_{j} \operatorname{div} v=0 .
$$

Differentiating and summing in $j$, we then conclude

$$
\frac{4}{3} \Delta \operatorname{div} v=0 \text {. }
$$

Thus div $v$ must be constant and, since any divergence has average zero, we conclude that $\operatorname{div} v=0$. Thus (146) implies that $\Delta v_{i}=0$ for every $i$, which in turn gives the desired conclusion.

From this observation, we conclude the identity

$$
\mathcal{S}(v)=\mathcal{R}(\operatorname{div} \mathcal{S}(v)) \quad \forall v \in C^{\infty}\left(\mathbb{T}^{3}, \mathbb{R}^{3}\right) .
$$

Indeed, first observe that $\mathcal{R}(z)=\mathcal{S}(w)$, where $w=\frac{1}{4} \mathcal{P}(u)+\frac{3}{4} u$ for the solution $u$ of $\Delta u=z-f z$ with $\int u=0$. Thus, applying the argument above, since both sides of (147) have zero averages, it suffices to show that they have the same divergence. But since $\operatorname{div} \mathcal{R}(z)=z-f z$, the divergences of the two sides of (147) obviously coincide.

Step 2. Next, for $a \in C^{\infty}\left(\mathbb{T}^{3}, \mathbb{R}^{3}\right), k \in \mathbb{Z}^{3} \backslash\{0\}$ and $\lambda \in \mathbb{N} \backslash\{0\}$, consider

$$
\mathscr{S}\left(a e^{i \lambda k \cdot x}\right):=-\mathcal{S}\left(\frac{3}{4} \frac{a}{\lambda^{2}|k|^{2}} e^{i \lambda k \cdot x}+\frac{1}{4 \lambda^{2}|k|^{2}}\left(a-\frac{(a \cdot k) k}{|k|^{2}}\right) e^{i \lambda k \cdot x}\right) .
$$

Observe that

$$
\mathscr{S}\left(b a e^{i \lambda k \cdot x}\right)-b \mathscr{S}\left(a e^{i \lambda k \cdot x}\right)=\frac{a A(b)}{\lambda^{2}} e^{i \lambda k \cdot x},
$$

where $A$ is an homogeneous differential operator of order one with constant coefficients (all depending only on $k$ ). Moreover,

$$
a e^{i \lambda k \cdot x}-\operatorname{div} \mathscr{S}\left(a e^{i \lambda k \cdot x}\right)=\frac{B_{1}(a)}{\lambda} e^{i \lambda k \cdot x}+\frac{B_{2}(a)}{\lambda^{2}} e^{i \lambda k \cdot x},
$$

where $B_{1}$ and $B_{2}$ are homogeneous differential operators of order 1 and 2 (respectively) with constant coefficients (again all depending only on $k$ ).

We use then the identity (147) to write

$$
\begin{aligned}
-[b, \mathcal{R}](F)= & \mathcal{R}(b F)-b \mathcal{R}(F)=\mathscr{S}\left(b a e^{i \lambda k \cdot x}\right)-b \mathscr{S}\left(a e^{i \lambda k \cdot x}\right) \\
& +\mathcal{R}\left(b F-\operatorname{div} \mathscr{S}\left(b a e^{i \lambda k \cdot x}\right)\right)-b \mathcal{R}\left(F-\operatorname{div} \mathscr{S}\left(a e^{i \lambda k \cdot x}\right)\right) \\
= & \frac{a A(b)}{\lambda^{2}} e^{i \lambda k \cdot x}+\mathcal{R}\left(\frac{B_{1}(a b)}{\lambda} e^{i \lambda k \cdot x}+\frac{B_{2}(a b)}{\lambda^{2}} e^{i \lambda k \cdot x}\right) \\
& -b \mathcal{R}\left(\frac{B_{1}(a)}{\lambda} e^{i \lambda k \cdot x}+\frac{B_{2}(a)}{\lambda^{2}} e^{i \lambda k \cdot x}\right) .
\end{aligned}
$$

Using the Leibniz rule we can write $B_{1}(a b)=B_{1}(a) b+a B_{1}(b)$ and $B_{2}(a b)=$ $B_{2}(a) b+a B_{2}(b)+C_{1}(a) C_{1}(b)$, where $C_{1}$ is an homogeneous operator of order 1. 
We can then reorder all terms to write

$$
\begin{aligned}
-[b, \mathcal{R}](F)= & \frac{a A(b)}{\lambda^{2}} e^{i \lambda k \cdot x} \\
& +\mathcal{R}\left(\frac{a B_{1}(b)}{\lambda} e^{i \lambda k \cdot x}\right)+\mathcal{R}\left(\frac{a B_{2}(b)+C_{1}(a) C_{1}(b)}{\lambda^{2}} e^{i \lambda k \cdot x}\right) \\
& -\frac{1}{\lambda}[b, \mathcal{R}]\left(B_{1}(a) e^{i \lambda k \cdot x}\right)-\frac{1}{\lambda^{2}}[b, \mathcal{R}]\left(B_{2}(a) e^{i \lambda k \cdot x}\right) .
\end{aligned}
$$

In the first three summands only derivatives of $b$ appear, but there are no zero order terms in $b$. We can then estimate the two terms in the second line applying Proposition G.1, with $m=N-1$ to the first summand and with $m=N-2$ to the second summand. Applying in addition interpolation identities, we conclude

$$
\begin{aligned}
\|[b, \mathcal{R}](F)\|_{\alpha} \leq & C \frac{\|a\|_{0}\|b\|_{1}}{\lambda^{2-\alpha}}+C \frac{\|a\|_{N-1+\alpha}\|b\|_{1+\alpha}+\|a\|_{N-2+\alpha}\|b\|_{2+\alpha}}{\lambda^{N-\alpha}} \\
& +C \frac{\|a\|_{1+\alpha}\|b\|_{N-1+\alpha}+\|a\|_{\alpha}\|b\|_{N+\alpha}}{\lambda^{N-\alpha}} \\
& +\frac{1}{\lambda} \underbrace{\left\|[b, \mathcal{R}]\left(B_{1}(a) e^{i \lambda k \cdot x}\right)\right\|_{\alpha}}_{I I}+\frac{1}{\lambda^{2}}\left\|[b, \mathcal{R}]\left(B_{2}(a) e^{i \lambda k \cdot x}\right)\right\|_{\alpha} .
\end{aligned}
$$

(Indeed, the above estimate is slightly sub-optimal up to fractional derivatives of order $\alpha$ and multiplying factors of order $\lambda^{\alpha}$.)

Step 3. We can now apply the same idea to the term $I I$ in (151), which is of the form $\left\|[b, \mathcal{R}]\left(F^{\prime}\right)\right\|_{\alpha}$, where $F^{\prime}(x)=B_{1}(a)(x) e^{i \lambda k \cdot x}$ and $B_{1}(a)$ are linear combinations of first order derivatives of $a$. However, this time we apply it with $N-1$ in place of $N$ and we estimate

$$
\begin{aligned}
\|[b, \mathcal{R}](F)\|_{\alpha} \leq & C \lambda^{\alpha-2}\|b\|_{1}\left(\|a\|_{0}+\lambda^{-1}\|a\|_{1}\right) \\
& +C \frac{\|a\|_{N-1+\alpha}\|b\|_{1+\alpha}+\|a\|_{N-2+\alpha}\|b\|_{2+\alpha}}{\lambda^{N-\alpha}} \\
& +C \frac{\|a\|_{2+\alpha}\|b\|_{N-2+\alpha}+\|a\|_{1+\alpha}\|b\|_{N-1+\alpha}+\|a\|_{\alpha}\|b\|_{N+\alpha}}{\lambda^{N-\alpha}} \\
& +\frac{1}{\lambda^{2}}\left\|[b, \mathcal{R}]\left(B_{2}^{\prime}(a) e^{i \lambda k \cdot x}\right)\right\|_{\alpha}+\frac{1}{\lambda^{3}}\left\|[b, \mathcal{R}]\left(B_{3}^{\prime}(a) e^{i \lambda k \cdot x}\right)\right\|_{\alpha},
\end{aligned}
$$

where $B_{2}^{\prime}=B_{2}+B_{1} \circ B_{1}$ is a second order operator and $B_{3}^{\prime}=B_{2} \circ B_{1}$ a third order operator (both with constant coefficients). Proceeding inductively now, 
we end up with

$$
\begin{aligned}
\|[b, \mathcal{R}](F)\|_{\alpha} \leq & C \lambda^{\alpha-2}\|b\|_{1} \sum_{i=1}^{N-2} \lambda^{-i}\|a\|_{i}+C \lambda^{\alpha-N} \sum_{i=0}^{N-1}\|a\|_{i+\alpha}\|b\|_{N-i+\alpha} \\
& +\frac{1}{\lambda^{N-1}}\left\|[b, \mathcal{R}]\left(B_{N-1}^{\prime}(a) e^{i \lambda k \cdot x}\right)\right\|_{\alpha} \\
& +\frac{1}{\lambda^{N}}\left\|[b, \mathcal{R}]\left(B_{N}^{\prime}(a) e^{i \lambda k \cdot x}\right)\right\|_{\alpha},
\end{aligned}
$$

where $B_{N-1}^{\prime}$ and $B_{N}^{\prime}$ are two linear constant coefficients operators of order $N-1$ and $N$ respectively.

Finally, we apply Proposition G.1 and Proposition F.1 to the final two terms and interpolate to reach the desired estimate.

To compare with the proof in [18], estimates analogous to Propositions F.1, G.1 and H.1 are established through a different approach in Sections 6 and 26 of [18], but there is also an additional section (Section 27) devoted to special solutions of $\operatorname{div} R=U$ obtained by solving a transport equation. This section corresponds, roughly speaking, to an improved estimate for the final term in (153). A similar improvement can be reached by observing that, since $[b, \mathcal{R}]=0$ when $b$ is a constant, we can assume, without loss of generality, that $b$ has average 0 , from which we conclude the control $\|b\|_{r} \leq C(r)\|\nabla b\|_{r-1}$ for all $r \geq 1$. Thus we could replace all terms $\|b\|_{r}$ with $\|\nabla b\|_{r-1}$ in (144). Since Proposition H.1 is applied to $b=v_{\ell}^{i}$ (i.e., components of $v_{\ell}$ ), the corresponding error estimates display a dependence upon $\nabla v$ rather than upon $v$ itself, which is natural in view of the Galilean invariance of the Euler equations (cf. [18]).

We do not keep track of this because it does not improve the main results of the paper.

\section{References}

[1] T. Buckmaster, Onsager's conjecture almost everywhere in time, Comm. Math. Phys. 333 (2015), 24pp., published online 7 January 2015. http://dx.doi.org/10. 1007/s00220-014-2262-z.

[2] A. Cheskidov, P. Constantin, S. Friedlander, and R. Shvydkoy, Energy conservation and Onsager's conjecture for the Euler equations, Nonlinearity 21 (2008), 1233-1252. MR 2422377. Zbl 1138.76020. http://dx.doi.org/10.1088/ 0951-7715/21/6/005.

[3] P. Constantin, W. E, and E. S. Titi, Onsager's conjecture on the energy conservation for solutions of Euler's equation, Comm. Math. Phys. 165 (1994), 207-209. MR 1298949. Zbl 0818.35085. http://dx.doi.org/10.1007/BF02099744.

[4] S. Conti, C. De Lellis, and L. Székelyhidi, JR., $h$-principle and rigidity for $C^{1, \alpha}$ isometric embeddings, in Nonlinear Partial Differential Equations, Abel Symposia 7, Springer-Verlag, New York, 2012, pp. 83-116. Zbl 1255.53038. 
[5] R. Courant, K. Friedrichs, and H. Lewy, On the partial difference equations of mathematical physics, IBM J. Res. Develop. 11 (1967), 215-234. MR 0213764. Zbl 0145.40402. http://dx.doi.org/10.1147/rd.112.0215.

[6] S. DANERI, Cauchy problem for dissipative Hölder solutions to the incompressible Euler equations, Comm. Math. Phys. 329 (2014), 745786. Zbl 1298.35140. http: //dx.doi.org/10.1007/s00220-014-1973-5.

[7] C. De Lellis and L. SzÉKelyhidi, JR., Dissipative Euler flows and Onsager's conjecture, pp. 1467-1505. MR 3254331. Zbl 06353607. http://dx.doi.org/10. 4171/JEMS/466.

[8] C. De Lellis and L. SzéKelyhidi, JR., The Euler equations as a differential inclusion, Ann. of Math. 170 (2009), 1417-1436. MR 2600877. Zbl 05710190. http://dx.doi.org/10.4007/annals.2009.170.1417.

[9] C. De Lellis and L. SzéKelyhidi, JR., On admissibility criteria for weak solutions of the Euler equations, Arch. Ration. Mech. Anal. 195 (2010), 225-260. MR 2564474. Zbl 1192.35138. http://dx.doi.org/10.1007/s00205-008-0201-x.

[10] C. De Lellis and L. Székelyhidi, JR., The $h$-principle and the equations of fluid dynamics, Bull. Amer. Math. Soc. 49 (2012), 347-375. MR 2917063. Zbl 1254.35180. http://dx.doi.org/10.1090/S0273-0979-2012-01376-9.

[11] C. De Lellis and L. SzÉKelyhidi, JR., Dissipative continuous Euler flows, Invent. Math. 193 (2013), 377-407. MR 3090182. Zbl 1280.35103. http://dx. doi.org/10.1007/s00222-012-0429-9.

[12] J. Duchon and R. Robert, Inertial energy dissipation for weak solutions of incompressible Euler and Navier-Stokes equations, Nonlinearity 13 (2000), 249255. MR 1734632. Zbl 1009.35062. http://dx.doi.org/10.1088/0951-7715/13/1/ 312 .

[13] G. L. Eyink, Energy dissipation without viscosity in ideal hydrodynamics. I. Fourier analysis and local energy transfer, Phys. D 78 (1994), 222-240. MR 1302409. Zbl 0817.76011. http://dx.doi.org/10.1016/0167-2789(94)90117-1.

[14] G. L. Eyink and K. R. Sreenivasan, Onsager and the theory of hydrodynamic turbulence, Rev. Modern Phys. 78 (2006), 87-135. MR 2214822. Zbl 1205.01032. http://dx.doi.org/10.1103/RevModPhys.78.87.

[15] U. Frisch, Turbulence, The legacy of A. N. Kolmogorov, Cambridge Univ. Press, Cambridge, 1995. MR 1428905. Zbl 0832.76001.

[16] D. Gilbarg and N. S. Trudinger, Elliptic Partial Differential Equations of Second Order, Classics in Math., Springer-Verlag, New York, 2001, reprint of the 1998 edition. MR 1814364. Zbl 1042.35002.

[17] M. Gromov, Partial Differential Relations, Ergeb. Math. Grenzgeb. 9, SpringerVerlag, New York, 1986. MR 0864505. Zbl 0651.53001. http://dx.doi.org/10. 1007/978-3-662-02267-2.

[18] P. Isett, Hölder continuous Euler flows in three dimensions with compact support in time, 2012, preprint.

[19] A. N. Kolmogorov, The local structure of turbulence in incompressible viscous fluid for very large Reynolds numbers, Proc. Roy. Soc. London Ser. A 434 
(1991), 9-13, translated from the Russian by V. Levin, Turbulence and stochastic processes: Kolmogorov's ideas 50 years on. MR 1124922. Zbl 1142.76389. http://dx.doi.org/10.1098/rspa.1991.0075.

[20] J. NAsh, $C^{1}$ isometric imbeddings, Ann. of Math. 60 (1954), 383-396. MR 0065993. Zbl 0058.37703. http://dx.doi.org/10.2307/1969840.

[21] L. Onsager, Statistical hydrodynamics, Nuovo Cimento 6 (1949), 279-287. MR 0036116.

[22] R. Robert, Statistical hydrodynamics (Onsager revisited), in Handbook of Mathematical Fluid Dynamics, Vol. II, North-Holland, Amsterdam, 2003, pp. 1-54. MR 1983588. http://dx.doi.org/10.1016/S1874-5792(03)80003-4.

[23] V. Scheffer, An inviscid flow with compact support in space-time, J. Geom. Anal. 3 (1993), 343-401. MR 1231007. Zbl 0836.76017. http://dx.doi.org/10. $1007 / \mathrm{BF} 02921318$.

[24] A. Shnirelman, Weak solutions with decreasing energy of incompressible Euler equations, Comm. Math. Phys. 210 (2000), 541-603. MR 1777341. Zbl 1011. 35107. http://dx.doi.org/10.1007/s002200050791.

(Received: August 8, 2013)

(Revised: August 19, 2014)

Institut für Mathematik, Universität Leipzig, Leipzig, Germany

E-mail: tristan.buckmaster@math.uni-leipzig.de

Current address: Courant Institute of Mathematical Sciences, New York University, New York, NY

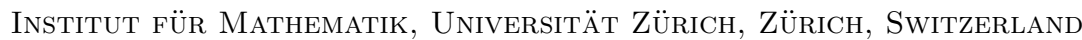

E-mail : camillo.delellis@math.uzh.ch

Department of Mathematics, Princeton University, Princeton, NJ

E-mail: pisett@math.princeton.edu

Current address: Department of Mathematics, Mit, Cambridge, MA

Institut Für Mathematik, Universität LeIPZIG, LeIPZIG, Germany

E-mail: laszlo.szekelyhidi@math.uni-leipzig.de 